\title{
$\checkmark$ Research Square \\ Intercellular exchange of Wnt ligands reduces cell population heterogeneity in embryogenesis
}

\section{Yudai Hatakeyama}

National Institute for Basic Biology

\section{Nen Saito}

Exploratory Research Center on Life and Living Systems https://orcid.org/0000-0002-8317-9389

\section{Yusuke Mii}

National Institute for Basic Biology and Okazaki Institute for Integrative Bioscience

https://orcid.org/0000-0002-1907-5665

\section{Takuma Shinozuka}

National Institute for Basic Biology

Tatsuya Takemoto

Tokushima University https://orcid.org/0000-0003-1860-0269

Honda Naoki

Hiroshima University

Shinji Takada ( $\sim$ stakada@nibb.ac.jp )

Exploratory Research Center on Life and Living Systems https://orcid.org/0000-0003-4125-6056

\section{Article}

Keywords: Wnt, epiblast, tailbud, NMP, paracrine, community effect, retinoic acid

Posted Date: February 2nd, 2022

DOI: https://doi.org/10.21203/rs.3.rs-1271602/v1

License: (c) (1) This work is licensed under a Creative Commons Attribution 4.0 International License.

Read Full License 


\title{
Intercellular exchange of Wnt ligands reduces cell population heterogeneity in embryogenesis
}

\author{
Yudai Hatakeyama ${ }^{1,2,3}$, Nen Saito ${ }^{1,2,3}$, Yusuke Mii ${ }^{1,2,3,4}$, Takuma Shinozuka ${ }^{1,2,3, \#}$, Tatsuya \\ Takemoto $^{5}$, Honda Naoki ${ }^{1,6}$, \& Shinji Takada ${ }^{1,2,3, *}$,
}

1 National Institute for Basic Biology and Exploratory Research Center on Life and Living Systems (ExCELLS), National Institutes of Natural Sciences, 5-1 Higashiyama, Myodaiji-cho, Okazaki, Aichi 444-8787, Japan

2 National Institute for Basic Biology, National Institutes of Natural Sciences, 5-1 Higashiyama, Myodaiji-cho, Okazaki, Aichi 444-8787, Japan

3 The Graduate University for Advanced Studies (SOKENDAI), 5-1 Higashiyama, Myodaijicho, Okazaki, Aichi 444-8787, Japan

4 PREST, Japan Science and Technology Agency (JST), Kawaguchi, Saitama, 332-0012, Japan

5 Institute of Advanced Medical Sciences, Tokushima University, 3-18-5 Kuramoto-cho,

Tokushima, 770-8503, Japan

6 Graduate School of Integrated Sciences for Life, Hiroshima University, 1-3-2 Kagamiyama, Higashi-hiroshima, Hiroshima, 739-8511, Japan

\# Present address: Nara Institute of Science and Technology, 8916-5 Takayama-cho, Ikoma, Nara 630-0912, Japan

*To whom correspondence should be addressed.

Shinji Takada Ph. D.; e-mail: stakada@nibb.ac.jp

Running title: Compensation of heterogeneity by paracrine Wnt signal

Key words: Wnt, epiblast, tailbud, NMP, paracrine, community effect, retinoic acid 


\begin{abstract}
Wnt signaling is required to maintain bipotent progenitors for neural and paraxial mesoderm cells, the neuromesodermal progenitor (NMP) cells that reside in the epiblast and tailbud. Since epiblast/tailbud cells receive Wnt ligands produced by one another, this exchange may average out the heterogeneity of Wnt signaling levels among these cells. Here, we examined this possibility by replacing endogenous Wnt3a with a receptor-fused form that can activate signaling in producing cells, but not in neighboring cells. Mutant mouse embryos showed a unique phenotype in which maintenance of many NMP cells was impaired, although some cells persisted for long periods. The epiblast cell population of these embryos increased heterogeneity in Wnt signaling levels as embryogenesis progressed and were sensitive to retinoic acid, an endogenous antagonist of NMP maintenance. Thus, mutual intercellular exchange of Wnt ligands in the epiblast cell population reduces heterogeneity and achieves robustness to environmental stress.
\end{abstract}

\title{
Main
}

The number of stem and progenitor cells is tightly controlled during embryogenesis and homeostasis. As the developmental context or external environment surrounding these cells changes, stem and progenitor cell populations respond to these changes, sometimes robustly, sometimes flexibly, thereby keeping these cells under control. In many cases, secreted signal proteins control maintenance and differentiation of stem/progenitor cells. However, mechanisms by which such signal proteins contribute to the robustness of these cell populations remain to be determined.

The body axis of vertebrate embryos elongates in an anterior-to-posterior fashion. During this elongation process, cells that constitute tissues in the trunk and tail are continuously generated from progenitor cells ${ }^{1}$. These progenitor cells are found in an area at the posterior end of embryos, termed the epiblast in early embryonic stages and the tail bud in later stages. Clonal lineage analysis revealed that both neural and paraxial mesodermal cell types are commonly generated from the same progenitor cells throughout the period of axis elongation ${ }^{2}$. These bipotent progenitor cells are called "Neuromesodermal Progenitor" (NMP) cells. NMP cells appear just before the onset of somitogenesis and are maintained until the conclusion of axis elongation. In mouse embryos, NMPs are located in the caudal lateral epiblast (CLE) posterior to the node-streak border of the primitive streak region and the chordoneural hinge $(\mathrm{CNH})$ of the tail bud ${ }^{2-7}$. Population and clonal analyses indicate that these cells behave like stem cells ${ }^{3,4}$. 
Cell signaling molecules and transcription factors are implicated in regulation of axis elongation, probably by maintaining NMPs. For instance, in the mouse, at least three Wnt ligands are sequentially expressed in the epiblast and tailbud. Wnt 3 expression is first activated in the posterior epiblast at E5.5, followed by Wnt $8 a$ and $W n t 3 a$ expression ${ }^{8-11}$, While Wnt3 and $W n t 8 a$ expression cease by early somite stage, $W n t 3 a$ expression continues until E12.5, when tail elongation is almost completed ${ }^{12}$. Along with expression of these Wnt ligands, a T-box transcription factor, $\mathrm{T} /$ Brachyury (Bra), is continuously expressed in the same region from the onset to the end of Wnt 3 expression ${ }^{13}$. Evidence suggests that Wnt signaling and Bra are important for maintenance of NMPs. Genetic studies of null mutant embryos of Wnt3a and Bra, showed their importance for axis elongation ${ }^{8,14}$, and lineage tracing of cells that express $\mathrm{Bra}$ revealed that both neural and paraxial mesoderm cells are derived from Bra-expressing cells ${ }^{15-}$ ${ }^{18}$. In addition to NMP maintenance, Wnt signaling and Bra are involved in fate determination between the neural and paraxial-mesodermal lineages ${ }^{14,19}$. Of note, Wnt signaling directly activates $B r a$ expression through Tcf transcription factor, while Bra is required for Wnt $3 a$ expression ${ }^{14,20-22}$. Thus, Wnt and Bra form a positive feedback loop in which each actively regulates expression of the other in NMP maintenance. Similarly, positive feedback between wnt 8 and tbxta (ntl), a zebrafish ortholog of Bra, has been reported in zebrafish ${ }^{23,24}$.

During maintenance of NMPs, activation of Wnt signaling and expression of Bra overlap widely in the epiblast and the tail bud, including the area where NMPs exists. Thus, some Wnt ligands may act in an autocrine manner in the epiblast and the tail bud, resulting in selfactivation of a Wnt/Bra regulatory loop in each cell. On the other hand, given that cells adjoining NMP cells also express Wnt ligands and Bra, paracrine Wnt ligands supplied by neighboring cells may also be involved in NMP maintenance. To examine the importance of Wnt paracrine function in maintenance of NMPs, we generated knock-in mouse embryos in which endogenous Wnt3a is replaced with a receptor-fused form that lacks paracrine activity, but maintains autocrine activity. Exacting analysis of Wnt paracrine-deficient embryos revealed the significance of the paracrine signal for maintenance of the NMP cell population and resilience to stress from external tissue. To the best of our knowledge, this is the first direct experimental evidence of the significance of intercellular exchange of secreted signal proteins in the emergence of cell population features. 


\section{Results}

\section{Wnt3a fused with Frizzled possesses signaling activity, but no paracrine activity}

To eliminate paracrine activity of WNT3A, we fused mouse WNT3A to the N-terminus of human FRIZZLED5 via 2 MYC tags (WNT3A-FZD5; Fig. 1a). Activity of WNT3A-FZD5 was examined in cells stably expressing TOP-FLASH reporter (STF293 cells) in comparison with authentic WNT3A, as well as GFP-fused WNT3A (GFP-WNT3A; Fig. 1a). Activity of GFPWNT3A was lower than that of authentic WNT3A (Fig. 1b), but sufficient to replace endogenous Wnt3a in vivo ${ }^{25}$. WNT3A-FZD5 activated canonical Wnt signaling to almost same extent as authentic WNT3A and more strongly than GFP-WNT3A, $48 \mathrm{~h}$ after transfection, and this activity was nearly saturated even after longer incubation (Fig. 1b). In contrast, whereas Wnt activity was activated in STF293 cells co-cultured with cells expressing intact WNT3A or GFP-WNT3A, almost no activation was detected in co-culture with WNT3A-FZD5-expressing cells (Fig. 1c). Consistent with this result, WNT3A-FZD5 was not detected in culture supernatant (Extended Data Fig. 1). These results show that as expected, WNT3A-FZD5 possesses sufficient signaling activity, but almost no paracrine activity.

\section{Axis elongation is impaired, but partially maintained in $\mathrm{Wnt} 3 a-F z d 5$ homozygous embryos}

We next generated mouse embryos in which endogenous $W n t 3 a$ is substituted for $W n t 3 a-F z d 5$, using a CRISPR/Cas9-mediated knock-in approach (Extended Data Fig. 2a-f). Mice heterozygous for Wnt3a-Fzd5 were morphologically normal and fertile (Fig. 1d-j, Extended Data Fig. 2g-k). As expected, Wnt3a-Fzd5 exhibited an expression pattern identical to that of endogenous Wnt $3 a$ in these embryos (Fig. 1d-g, Extended Data Fig. 2g-j). In addition, Western blotting analysis revealed that $W n t 3 a-F z d 5$ heterozygous and homozygous embryos expressed WNT3A-FZD5 proteins at the expense of authentic Wnt3a in the posterior region (Fig. 1h). Thus, $W n t 3 a-F z d 5$ properly replaced endogenous $W n t 3 a$, being expressed in the same spatial pattern as endogenous $W n t 3 a$.

While $W n t 3 a-F z d 5$ heterozygotes $\left(W n t 3 a^{+/ F z d 5}\right.$ ) showed no obvious embryonic abnormality (Fig. 1i, j), adult $W n t 3 a-F z d 5$ homozygotes $\left(W n t 3 a^{F z d 5 / F z d 5}\right.$, were embryonically lethal and die after E12.5 (Extended Data Fig. 2k and data not shown). However, the phenotype of Wnt $3 a$ $F z d 5$ homozygotes was milder than that of Wnt $3 a$ null mutant embryos, which die around E9.5 with posterior truncation ${ }^{8}$. Thus, even though WNT3A-FZD5 has sufficient signaling activity in vitro (Fig.s 1b, c), Wnt3a-Fzd5 partially, but not completely, substitutes for endogenous Wnt $3 a$.

To better understand this puzzling phenotype of Wnt3a-Fzd5 homozygous embryos, we examined their morphology. While the gross morphology of Wnt3a-Fzd5 homozygous embryos 
appeared normal in the anterior trunk, it was highly disorganized posterior to the hindlimbs (Fig. 1i-k). This posterior defect became evident no later than E8.75 (Fig. 4a, b and 5k, 1). Transverse images of E11.5 embryos stained with DAPI showed that neural tube morphology was gradually disturbed along the anterior-posterior axis in these embryos (Fig. 11-n). This disruption was evident in the intermediate region between fore- and hindlimbs (Fig. 1n') and pronounced in the more posterior region, resulting in an opened neural tube at the hindlimb level (Fig. 1n"). However, in spite of this severe defect in posterior morphogenesis, a thin, kinked tail-like structure was found at the posterior end of these embryos (Fig. 1k: red arrowhead).

Whole mount in situ hybridization analyses also revealed that the neural tube, marked by Sox 2 expression, was abnormally opened posterior to the hindlimb at E10.5 in Wnt3a-Fzd5 homozygous embryos (Fig. 2r, s). Somites, stained with the Uncx 4.1 probe, were normally formed in the anterior trunk, but their size is reduced posterior to the hindlimb (Fig. $2 \mathrm{~m}, \mathrm{n}$ ). Of note, Brachyury (Bra), which is expressed in the tailbud and notochord of normal embryos, was expressed at the tip of the thin and kinked tail, although the number of Bra-positive cells was decreased (Fig. 2a, b). In addition, Tbx6, expression of which is turned on immediately after specification to the paraxial mesoderm lineage, was also expressed at this posterior end (Fig. $2 \mathrm{~g}$, h). Notably, expression of Bra and Tbx6 at the posterior tip was maintained even at E12.5, when tail elongation is nearly arrested in normal embryos (Fig. $2 \mathrm{w}$-ab) Thus, in Wnt $3 a-F z d 5$ homozygous embryos, trunk morphogenesis was disrupted at the hindlimb level, accompanied by reduction of tailbud size, but differentiation from the tailbud appears to be maintained throughout the period of axis elongation.

\section{Wnt-positive progenitor cells are responsible for abnormal neural and somite development in Wnt3a-Fzd5 homozygous embryos}

To examine the impact of tailbud reduction in posterior morphogenesis of $W n t 3 a-F z d 5$ homozygous embryos, we followed cells that had activated Wnt signaling, because Wnt signaling is activated in progenitor cells in the epiblast and tailbud region. To this end, Axin2creERT2 and floxed tdTomato alleles were introduced into Wnt $3 a-F z d 5$ homozygous embryos. Tamoxifen was injected into pregnant female mice at 7.5 or 8.5 days post coitus (dpc) and embryos were fixed at E10.5 (Fig. 3a and Extended Data Fig. 3a). In control, wild-type, and $W n t 3 a-F z d 5$ heterozygous, embryos, labelled cells were detected in most tissues at the hindlimb level, regardless of the timing of tamoxifen administration (Extended Data Fig. 3b-e). However, when tamoxifen was injected at $8.5 \mathrm{dpc}$, but not at E7.5 dpc, labelled cells were rarely detected in the ventral neural tube at the hindlimb level (Extended Data Fig. 3d, e), showing that the 
origin of ventral neural cells loses Wnt signaling after E7.5. As in control littermates, in Wnt3a$F z d 5$ homozygous embryos labelled at $8.5 \mathrm{dpc}$, labelled cells were similarly distributed in most tissues, except neural tube (Fig.s $3 b$ and c). Labelling efficiency monitored in somites (Fig. 3d) and the nephric duct (Fig. 3e) was not significantly changed between littermates. However, the number of labelled cells was specifically reduced in somites (Fig. 3f) and neural tube (Fig. 3g) in $W n t 3 a-F z d 5$ homozygous embryos. Thus, the number of cells derived from Wnt-positive progenitors at E8.5 was decreased in dorsal neural tube and somites of Wnt3a-Fzd5 homozygous embryos. It is plausible that the decrease of dorsal neural cells results in the opened neural tube in $W n t 3 a-F z d 5$ homozygous embryos.

As described above, Wnt3a is expressed in the roof plate of the neural tube, in addition to the epiblast and tailbud (Fig.s 1d-g). Thus, it also seems probable that $W n t 3 a-F z d 5$ expression in the roof plate region causes the morphological abnormality in $W n t 3 a-F z d 5$ homozygous embryos. To test this possibility, we examined the contribution of $\mathrm{Bra}$ to this phenotype, because $\mathrm{Bra}$ interacts specifically with $W n t 3 a$ in development of the epiblast/tailbud, but not the roof plate. While Wnt $3 a-F z 5$ heterozygotes (Extended Data Fig. 4a) and Bra single heterozygotes (Extended Data Fig. 4b) appeared normal, Wnt3a-Fz5 and Bra compound heterozygous

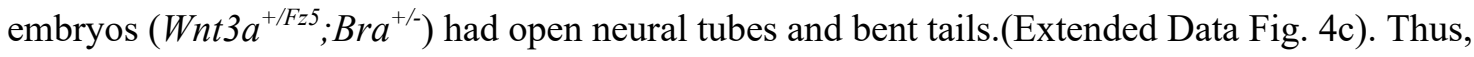
interaction of $W n t 3 a-F z 5$ and $B r a$ in the epiblast and tailbud region is responsible for the phenotype of Wnt3a-Fzd5 homozygous embryos.

\section{Evidence indicates that the phenotype of $W n t 3 a-F z d 5$ homozygotes is due to the lack of paracrine activity}

Reduction of the Wnt3a signal impairs maintenance of the tailbud, including NMP cells ${ }^{8,16}$. This defect results in truncation of A-P elongation in a manner dependent on Wnt3a activity ${ }^{26}$. Since $W n t 3 a^{v t /-}(v t /-)$ embryos, which possess one copy of a hypomorphic ( $\left.v t\right)$ allele of $W n t 3 a$ with reduced $W n t 3 a$ expression in the tailbud (Extended Data Fig. 5a-f: Greco et al., 1996) impairs trunk development at the same level as in Wnt3a-Fzd5 homozygous embryos (Fig. 2c, d, i, j, o, $\mathrm{p}$, t, and $\mathrm{u}$ ), we compared the morphology of $v t /$ - embryos with Wnt $3 a-F z d 5$ homozygous embryos. In contrast to $W n t 3 a-F z d 5$ homozygotes, $v t /$ - embryos did not exhibit thin, kinked tails and open neural tubes, but they failed to maintain the tailbud, marked by Bra and Tbx6 expression, at E10.5 (Fig. 2d, j) and E12.5 (Extended Data Fig. 5g, h). Therefore, the phenotype of Wnt3a-Fzd5 homozygotes is unique, compared with other Wnt3a hypomorphic mutants and is not simply due to decreased Wnt3a activity. 
This characteristic phenotype of $W n t 3 a-F z d 5$ homozygous embryos was also observed in $W n t 3 a^{F z d 5 /}$ embryos (Extended Data Fig. 6a-c, e-g). Because Wnt3a-Fzd5 heterozygous embryos $\left(W n t 3 a^{+/ F z d 5}\right)$ appeared normal, as previously described (Fig. 1j , Extended Data Fig. 6a, e), $W n t 3 a-F z d 5$ seems to cause this phenotype in the absence of wild-type Wnt3a. Furthermore, this phenotype can be rescued depending on the expression level of Wnt3a, because the phenotype of $W n t 3 a^{F z d 5 /-}$ embryos was partially rescued by replacing the null allele to $v t$ ( $W n t 3 a^{F z d 5 / v t}$; Extended Data Fig. 6d, h). Based on the results of these analyses using various Wnt3a mutants, the characteristic phenotype of $W n t 3 a-F z d 5$ homozygotes is due to some property lost in Wnt3a-Fzd5, most likely paracrine activity.

\section{NMP cells are reduced, but maintained in Wnt3a-Fzd5 homozygous embryos}

In spite of improper development in the posterior neural tube and somites, our analyses with molecular markers revealed that differentiation of paraxial mesoderm and neural cells was partially maintained in Wnt3a-Fzd5 homozygous embryos (Fig. 2b, h, n, s, x, aa). Furthermore, the tailbud marked by expression of $B r a$, was maintained at the posterior tip of the tail of these embryos(Fig. 2a, b, x, y, aa, ab). These data suggest that a small number of NMP cells persist in Wnt $3 a-F z d 5$ homozygous embryos.

Since one of the characteristics of NMP cells is expression of Bra and Sox ${ }^{27}$, we compared the number of Bra and Sox 2 double-positive cells using immunohistochemistry in Wnt3a-Fzd5 homozygous embryos and control littermates (Fig. 4a-x and Extended Data Fig. 7). The number of Bra and Sox2 double-positive cells started to diminish at E8.75 in Wnt3a-Fzd5 homozygous embryos (Fig. 4a-p), but a small number of double-positive cells were still maintained at E11.5 (Fig. 4q, r, u, v). In contrast, double-positive cells disappeared in vt/- embryos at E11.5 (Fig. 4s, $\mathrm{w}, \mathrm{t}, \mathrm{x})$. These results support the idea that a small number of NMP cells are specifically maintained in $W n t 3 a-F z d 5$ homozygous embryos, even after trunk development is impaired.

\section{Wnt signaling activity persists in a small number of epiblast cells in Wnt3a-Fzd5 homozygous embryos}

To further investigate the defect of $W n t 3 a-F z d 5$ homozygous embryos, we directly examined Wnt signaling activity in individual cells in the epiblast. To this end, we utilized the R26 $\mathrm{WntV}$ is reporter, expression of which is driven by heptameric TCF/LEF1 binding sequences combined with a viral minimal promoter in the Rosa26 locus ${ }^{28}$. This reporter responds in a graded fashion to a wide range of Wnt signal strengths. In addition, the histone H2B-EGFP protein, used as a fluorescent reporter, facilitates single-cell resolution analysis under confocal microscopy. In this study, fluorescence of this reporter was measured in individual cells in a 
photon-counting mode. Epiblast cells in the areas lateral to the node were individually analyzed in a single confocal plane.

During development of mouse epiblast, three Wnt ligands, Wnt3, Wnt8, and Wnt3a, sequentially activate Wnt signaling ${ }^{8,10,13,29}$. Because $W n t 3$ and $W n t 8$ are expressed prior to $W n t 3 a$, Wnt activity was detected even in Wnt $3 a$ null mutant embryos at early headfold (EHF) stage (E7.0; Takemoto et al., 2016). At this stage, no obvious change in Wnt activity was detected in Wnt $3 a-F z d 5$ homozygous embryos, as predicted (Fig. 5a-c). Then, Wnt3a expression was activated, and Wnt signaling level subsequently increased in both control and Wnt3a-Fzd5 homozygous embryos (Fig. 5d, e and Extended Data Fig. 8a), but not in Wnt3a null embryos (Extended Data Fig. 8b), at the late headfold (LHF) stage (E7.5). Thus, Wnt signaling was properly activated in the initial phase of Wnt3a-dependent activation, even in Wnt $3 a-F z d 5$ homozygous embryos. Of note, in these stages, the level of the fluorescent reporter differed among epiblast cells in both control and Wnt3a-Fzd5 homozygous embryos.

From early somite stage (E8.0), Wnt signaling began to be perturbed in Wnt3a-Fzd5 homozygous embryos. At E8.0, around 2-3-somite stage, the number of Wnt signaling-positive cells was reduced in the anterior and lateral epiblast regions of Wnt3a-Fzd5 homozygous embryos (Fig. 5f, g, h, o). The reduction in Wnt signaling-positive cells was pronounced in most of the epiblast region of Wnt3a-Fzd5 homozygous embryos at E8.75, where Wnt-positive and negative cells were distributed in a patch-work pattern (Fig. $5 \mathrm{f}, \mathrm{k}, 1, \mathrm{p}$ ). This reduction was enhanced by E9.5, but some Wnt-positive cells remained at the posterior end of Wnt3a-Fzd5 homozygous embryos (Fig. 5q, r). These posterior Wnt-positive cells were further maintained until E11.5 (data not shown). On the other hand, in $v t$ /- embryos, the decrease of Wnt signaling started at E8.75 (Fig. 5i, j, m, n). In contrast to Wnt3a-Fzd5 homozygous embryos, Wnt signaling was almost abolished around E9.5 in epiblast (Fig. 5s, t). Furthermore, the activity level appeared to decrease gradually in most Wnt-positive epiblast cells and the deviation of Wnt-activity in epiblast population was smaller than in Wnt3a-Fzd5 homozygous embryos.

Taken together, in Wnt3a-Fzd5 homozygous embryos, reduction of Wnt activity occurred from early somite stage, but a small number of Wnt-positive cells remain longer. Notably, Wnt activity appeared to fluctuate between adjacent cells even in control embryos, but in Wnt3a$F z d 5$ homozygous embryos this heterogeneity was enhanced (Fig. 5g-p). Probably, the accelerated reduction of Wnt activity in many epiblast cells reduces the number of NMP cells, as well as of neural and somite cells produced by NMP cells in Wnt3a-Fzd5 homozygous embryos. In contrast, persistent activation of Wnt signaling in the other epiblast cells probably 
contributes to maintenance of a small number of NMP cells, resulting in formation of thin, kinked tails.

\section{Retinoic acid enhances the phenotype of Wnt3a-Fzd5 homozygous embryos}

Since impairment of Wnt activation was observed from early somite stage, it seems probable that somite formation affects the Wnt3a-Fzd5-specific reduction of Wnt-active cells. Interestingly, retinoic acid (RA), which is synthesized in somite cells, antagonizes the function of Bra in zebrafish embryos ${ }^{24}$. Consistently, mouse embryos with mutated cyp $26 a$, which encodes an enzyme to degrade RA, exhibit an axis truncation phenotype, similar to Wnt $3 a$ and Bra mutant embryos ${ }^{30}$. Thus, we hypothesized that epiblast cells of $W n t 3 a-F z d 5$ homozygous embryos are more sensitive to RA in maintenance of Wnt activity.

To address this issue, female $W n t 3 a-F z d 5$ heterozygous mice intercrossed with $W n t 3 a-F z d 5$ heterozygous males were treated with RA 7.5 days post coitum and effects of RA on the phonotype of mutant embryos were examined. RA treatment specifically enhanced the abnormality in gross morphology of Wnt3a-Fzd5 homozygous embryos (Fig. 6a-h). Furthermore, Wnt reporter analysis revealed that RA treatment enhanced the specifically reduced pattern of Wnt activity in Wnt3a-Fzd5 homozygous embryos, showing enhancement of a patch-work pattern, irrespective of cell position along the anterior-posterior axis (Fig. 6i-m). This result suggests that the epiblast cell population of $W n t 3 a-F z d 5$ homozygous embryos is specifically susceptible to RA.

\section{Mathematical modeling supports the importance of paracrine function in maintaining Wnt-positive epiblast cell populations}

The results described above strongly suggest that lack of paracrine signaling enhances heterogeneity of Wnt activity in the epiblast cell population and that a cell population with such enhanced heterogeneity is more sensitive to antagonists, like RA. Thus, we also tested the validity of these ideas by creating a mathematical model (Fig. 7a-g, Extended Data Fig. 9 and Movie1). In this model, spatiotemporal changes in Wnt activity were compared in a hypothetical epiblast plane with and without intercellular exchange of Wnt ligands. The temporal increase or decrease of Wnt activity in each cell is defined by the production rate regulated by autocatalysis, which represents a positive feedback loop of Wnt3a/Bra, in addition to the basic rate of production and degradation of Wnt ligands. The stochastic increase/decrease in Wnt activity is also incorporated as a noise term. In this virtual plane, we assume that each cell divides stochastically and that a newly produced daughter cell locates laterally or anteriorly to the original cell. It is also assumed that an RA gradient from anterior to posterior is imposed 
at a specific time, which represents anteroposterior diffusion of RA. However, in this virtual space, cells that are aligned along the left-right axis were treated as if there is no difference in their distance from the RA source (Fig. 7a).

By using adjusted parameters, we first simulated spatiotemporal patterns of Wnt activity in the hypothetical epiblast plane under conditions in which intercellular exchange of Wnt ligands is present (Fig. 7b, d, e) and absent (Fingers 7c, f). Wnt activity levels decreased after the addition of RA ( $t>0$ shown in Fig. 7g, Extended Data Fig. 9a , and movie Extended Data Movie1) and the number of Wnt-low cells increased in the absence of intercellular Wnt exchange. However, a small number of Wnt-high cells remained for a while (until $t=3$ shown in Fig. 7c, g, Extended Data Movie 1B). On the other hand, if the production rate was reduced, mimicking the situation of $v t$ /- embryos, Wnt-low cells gradually increased and few Wnt-high cell remained (Fig..7D, G and Extended Data Movie 1C). These simulations showed that a lack of intercellular Wnt exchange reproduced the spatio-temporal pattern of Wnt activity observed in Wnt3a-Fzd5 homozygous embryos. Furthermore, we reproduced the sensitivity of $W n t 3 a-F z d 5$ homozygote cells when the RA concentration was uniformly increased in the hypothetical epiblast plane (Fig. 7e, f, g, and Extended Data Movies 1d, e). Taken together, these simulations based on our mathematical model support the idea that the Wnt paracrine signal reduces heterogeneity in Wnt activity in the epiblast cell population and increases robustness to RA.

\section{Discussion}

It is widely believed that secreted signal proteins act on cells in the vicinity of the source cells, and in some cases, more distally ${ }^{31,32}$. In contrast, in the epiblast and tailbud, most cells both produce and receive Wnt ligands ${ }^{16,33-35}$. As a result, Wnt ligands secreted from each cell into the extracellular space activate the intracellular Wnt signaling pathway in cells in the population. As a result, Wnt3a ligands secreted extracellularly from each cell activate the intracellular Wnt signaling pathway in cells within the population. Thus, in contrast to unidirectional transfer from Wnt-producing cells to receiving cells, Wnt ligands seem to be reciprocally exchanged between epiblast and tailbud cells.

To understand the biological significance of reciprocal ligand exchange within a cell population, we generated Wnt3a-Fzd5 homozygous embryos, in which Wnt3a-mediated intercellular communication, or paracrine function, is specifically impaired. In these embryos, the number of Wnt-positive cells decreases rapidly from the anterior and lateral sides of the epiblast after RA begins to be synthesized in the somite, but a small number of Wnt-positive cells, including 
NMP cells, remain at the posterior end for a long time. Precise examination of $W n t 3 a-F z d 5$ homozygous embryos and mathematical simulation support a model in which Wnt3a-mediated intercellular communication is required for maintenance of the NMP population (Figure $7 \mathrm{~h}$ ).

In epiblast and tailbud regions, including NMPs, Wnt3a-expressing cells also express Bra ${ }^{14}$. Bra is a direct transcriptional target of the Wnt signaling pathway, whereas Wnt3a expression is also dependent on Bra ${ }^{14,18}$. Thus, Wnt3a and Bra mutually activate one another, forming a positive feedback regulatory loop. Because positive feedback amplifies small changes, this regulatory system can rapidly increase or decrease the amount of Wnt3a and Bra in a cell ${ }^{36}$.

It seems plausible that the epiblast/tailbud cells vary with respect to Wnt production and degradation rates, efficiency of feedback amplification, and/or resistance to environmental factors that reduce Wnt activity. Thus, cells that are prone to losing Wnt activity rapidly lose this activity due to the positive feedback, resulting in an increased disparity generated by the fluctuations. In the epiblast of $W n t 3 a-F z d 5$ homozygotes, the number of cells with little or no Wnt activity rapidly increases from E8.0. In contrast, a small, but significant number of cells maintain high Wnt activity for a long time in these embryos. A probable reason for persistence of Wnt-high cells is that the change of Wnt activity in these cells is below the threshold to trigger a rapid decrease by positive feedback. Actually, our mathematical model, which assumes fluctuation in Wnt activity and positive feedback regulation, produces similar spatial patterns of Wnt activity in $W n t 3 a-F z d 5$ homozygous cell populations under conditions without Wntmediated intercellular communication.

In contrast, in control embryos, including $W n t 3 a-F z d 5$ heterozygotes, the number of Wnt3a positive cells slowly decreased and disappeared around E13.5, when axis elongation was terminated. Probably, in these embryos, Wnt ligands supplied by neighboring Wnt-high cells compensate to some extent for the decrease of Wnt activity in Wnt-low cells. Thus, the exchange of Wnt3a ligands appears to compensate for the rapid decrease in Wnt activity in individual cells. Taken together, positive feedback regulation can amplify heterogeneity among members of the cell population, but our results suggest that sharing of intercellular components of the feedback loop, such as Wnt ligands, inhibits amplification of this heterogeneity.

In the epiblast of $W n t 3 a-F z d 5$ homozygotes, Wnt activity rapidly decreases in many cells at E8.0, when several anterior somites are generated. In these embryos, the decrease in Wnt activity was more pronounced anteriorly and laterally in the epiblast. Of note, in early mouse embryos, RA synthesis requires retinaldehyde dehydrogenase 2 (RALDH2/ALDH1a2), which is 
activated in somites and lateral plate mesoderm ${ }^{37,38}$. A line of evidence has shown that RA signaling antagonizes Wnt/Bra activity gradually from the anterior side of the epiblast and tailbud region in the mouse embryo $24,30,37,39,40$. Thus, we speculated that the epiblast cell population in Wnt3a-Fzd5 homozygotes is sensitive to RA stress originating from tissues developed anteriorly and laterally to the epiblast.

Actually, RA treatment reduces Wnt signaling in epiblast cells specifically in Wnt3a-Fzd5 homozygous embryos, indicating that the epiblast cell population in these embryos is more susceptible to RA. Probably due to a failure to reduce heterogeneity in these embryos, an RAtriggered decrease in Wnt activity may be amplified rapidly via a positive feedback loop in individual cells. Thus, maintaining cooperativity among members of the epiblast/tailbud cell population and reducing the disparity in Wnt signaling among members may render the cell population more resilient to external stress.

It has been shown that intercellular communication within a cell population is critical to regulate cell differentiation. In Xenopus gastrulas, muscle progenitor cells communicate with each other as they differentiate. In such a case, more than one hundred Xenopus muscle precursor cells transplanted into ectoderm sandwiches can differentiate, while smaller groups and single cells cannot ${ }^{41,42}$. This cell number-dependent differentiation was described as a "community effect." It is caused by an intercellular interaction among precursor cells and such an interaction is necessary for the cells to differentiate. Theoretical studies have suggested that the positive feedback mediated by intercellular communication is the mechanism underlying this cell number-dependent differentiation ${ }^{43}$.

In this study, we showed that Wnt-mediated intercellular communication is actually involved in maintenance of the cell population in the epiblast/tailbud region. In this case, intercellular exchange of Wnt ligands is important to compensate for the disparity amplified via positive feedback from Wnt3a and Bra. An interesting question is whether a similar molecular network is involved in other events in which a community effect is exerted. Differences in the efficiency of cell signaling or the amplification efficiency of positive feedback loops probably generate differences in the features of cell populations. If this is the case, it will be important in future studies to identify key parameters in the molecular network to produce each of these events. 


\section{Methods}

\section{Mice}

Animal care and experiments were performed in accordance with guidelines for animal experimentation of the National Institutes for Natural Sciences. All animal experiments were approved by the Animal Research Committee of National Institutes for Natural Sciences. Mice were maintained in a light- and temperature-controlled room using a $12 \mathrm{~h}: 12 \mathrm{~h}$ light:dark cycle at $21 \pm 2^{\circ} \mathrm{C}$. Embryos derived from timed matings were genotyped by PCR with DNA from yolk sacs or embryos. PCR conditions and primer sequences for $\mathrm{Wnt} 3 \mathrm{a} \mathrm{KO}^{8}$ and $\mathrm{Wnt}$ vis reporter ${ }^{28}$ mice have been previously described.

\section{Cell culture and transfection}

HEK293T cells and STF293 cells, which are HEK293 cells stably expressing Super 7x TOPFlash ${ }^{44}$, were cultured at $37^{\circ} \mathrm{C}$ in a 1:1 mixture of DMEM and Ham's F12 medium supplemented with $8.3 \%$ fetal bovine serum.

Plasmids were transfected into HEK 293T cells or STF293 cells using FuGENE6 transfection reagent (Roche). Culture medium was changed $6 \mathrm{~h}$ after transfection. At 24, 48, and $72 \mathrm{~h}$ after transfection, cells and culture medium were harvested for Western blotting and luciferase reporter assay. In co-culture experiments, HEK 293T cells transfected with each plasmid were collected $24 \mathrm{~h}$ after transfection and mixed 1:1 with STF293 cells. The luciferase reporter assay was performed 24 or $48 \mathrm{~h}$ after co-culture. Details of Western blotting and the luciferase reporter assay are described below.

\section{Plasmid construction}

To generate plasmid constructs from which Wnt3a fused with human Frizzled $5(\mathrm{hFzd} 5)$ is expressed, a DNA fragment encoding the full length of mouse Wnt3a protein fused to the Nterminus of hFzd5 mediated with 2xMyc tag (TSEQKLISEEDLNEMEQKLISEEDLRS) (Extended Data Fig.1a), was integrated between the ClaI and XbaI sites of pCSf107 plasmid vector, which carries the CMV IE94 promoter. This fusion protein was designed to remove the signal peptide of $\mathrm{hFz} 5$, resulting in direct fusion of the $\mathrm{N}$-terminus of $\mathrm{hFzd} 5$ to the $2 \mathrm{xMyc}$ tag. DNA encoding the full length of mouse Wnt3a and EGFP fused Wnt3a(GFP-Wnt3a) was integrated into pCS2 plasmid vector.

\section{Western blotting and luciferase reporter assay}

For detection of proteins in cultured cells and culture supernatant, samples were collected at the time points described in "Cell Culture and Transfection." To detect Wnt3a-Fzd5 proteins in 
embryos, the area posterior to the newly formed somite of E8.5 embryos was cut and collected. SDS-PAGE was carried out according to a standard protocol ${ }^{45}$. Briefly, each sample was mixed with 2x sample buffer [4\% SDS, 20\% glycerol, $0.001 \%$ bromophenol blue and $0.125 \mathrm{M}$ Tris $\mathrm{HCl}(\mathrm{pH} \mathrm{6.8)}]$ and heated at $37^{\circ} \mathrm{C}$ for $1 \mathrm{~h}$. Samples were electrophoresed using $10 \%$ polyacrylamide gels. After electroporation, proteins on the gel were transferred to a polyvinylidene difluoride (PVDF) membrane (Millipore). These membranes were treated overnight at $4{ }^{\circ} \mathrm{C}$ with primary antibody (mouse anti-mouse Wnt3a antibody: Takada et al., Dev. Cell, 2006), followed by treatment with secondary antibodies (goat anti-mouse IgG : HRP conjugated, Promega W402B) for $1 \mathrm{~h}$ at room temperature. Finally, these proteins were visualized using an Enhanced Chemiluminescent Detection System (Amersham).

Luciferase reporter assay was performed according to the manufacturer's protocol (Dual-Glo Luciferase Assay System: Promega). Since STF293 cells contain a firefly Luciferase cDNA driven by eight tandem repeats of the TCF binding site, Wnt activity was quantified by monitoring activity of firefly Luciferase. Therefore, Luciferase is expressed depending on the strength of Wnt signaling. Renilla luciferase was used as an internal control to compensate for the mosaic nature of gene transfection. The activity of Luciferase was detected using a Luminometer (Turner Designs).

\section{Generation of $W n t 3 a-F z d 5$ knock-in mice}

In Wnt3a-Fzd5 knock-in mice, a DNA fragment encoding the MYC-hFZD5 fragment was designed to be integrated just before the stop codon in exon 4 of the mouse Wnt $3 a$ gene (Extended Data Fig. 1). The resulting protein expressed from this recombined locus is the same as that expressed in the cell culture experiment described above. To generate this knock-in allele, a pLSODN-3-based plasmid containing a DNA fragment of $m y c-F z d 5$ was co-injected with plasmids to express gRNA and Cas9 in fertilized eggs. The sequence of the gRNA is as follows: 5'-TTAGGAGCTCTCCTACTTGC-3'. This gRNA was inserted into pX330.

Genotyping was carried out by PCR using the following primers:

Wnt3a-Fzd5 5F, 5'-TGGTGCTTATCTGCCATTCC-3';

Wnt3a-Fzd5 WTF2, 5'-GTCACATGCACCTCAAGTGC-3';

Wnt3a-Fzd5 7F, 5'-GGTGTGCCAGGAAATCACGG-3';

Wnt3a-Fzd5 7R, 5'-GGACACCTGCTTGTGGTAGG-3';

Wnt3a-Fzd5 WTR2, 5'-AGGATCCTTCCTAGCAGTCC-3';

Wnt3a-Fzd5 4R, 5'-TTTCTACAGTTGACCGGCTC-3'.

The combination of primers used for PCR is shown in Extended Data Fig. 1. Fragments of 2,564-bp and 3,338-bp were expected from the 5'-region of wild-type Wnt3a and Wnt3a-Fzd5 
alleles, respectively. On the other hand, a 2,368-bp fragment and a 3,564-bp fragment were expected in the 3'-region of wild-type Wnt3a and Wnt3a-Fzd5 alleles, respectively.

\section{In situ hybridization}

Whole-mount in situ hybridization was performed using digoxigenin-labeled, antisense RNA probes. Briefly, embryos collected at the indicated stages were fixed with $4 \%$ paraformadyhyde (PFA) overnight at $4^{\circ} \mathrm{C}$, washed with PBS, and treated with $20 \mu \mathrm{g} / \mathrm{mL}$ of proteinase $\mathrm{K}$ for 5 $\min$. These embryos were incubated in hybridization buffer ( $50 \%$ formamide, $5 \times \mathrm{SSC}, 1 \% \mathrm{SDS}$, $50 \mu \mathrm{g} / \mathrm{mL}$ tRNA) overnight at $55^{\circ} \mathrm{C}$. The next day, embryos were washed consecutively with $5 \times$ SSC, $2 \times$ SSC, and Tris-buffered saline with Tween 20 (TBST). Next, embryos were incubated with 1\% sheep serum (Sigma) in TBST for $1 \mathrm{~h}$ and then treated with a 1:500 dilution of antidigoxigenin-AP Fab fragments (Roche) overnight at $4^{\circ} \mathrm{C}$. The following day, embryos were washed with TBST and alkaline phosphatase buffer $[100 \mathrm{mM} \mathrm{NaCl}, 100 \mathrm{mM}$ Tris-HCl (pH 9.5), $50 \mathrm{mM} \mathrm{MgCl} 2,0.5 \%$ Tween 20], and signals were developed using BM Purple (Roche). Wild-type and mutant embryos were stained for the same period in individual experiments. The following probes that have been reported previously were used: mouse $W n t 3 a^{46}$, mouse Brachyury and mouse Tbx $6^{14}$, mouse Uncx $4.1^{47}$, and human FRIZZLED $5^{48}$. To generate a Sox 2 probe, the first exon of mouse Sox2 was amplified from mouse genomic DNA and cloned to generate an antisense probe.

\section{Immunofluorescence}

Whole-mount immunofluorescence was performed on embryos collected at the indicated stages. These embryos were fixed with $4 \%$ PFA overnight at $4^{\circ} \mathrm{C}$, and washed with PBS. Embryos were incubated overnight at $4{ }^{\circ} \mathrm{C}$ with the following primary antibodies: rabbit anti-Sox2 (polyclonal, Millipore, AB5603, 1:200) and goat anti-Brachyury (polyclonal, Santacruz, 17745, 1:1000). After washing with PBS, embryos were incubated overnight at $4^{\circ} \mathrm{C}$ with DAPI (Dojindo) and following secondary antibodies: donkey anti-rabbit IgG (Alexa fluor 647 conjugated, 1:500) and donkey anti-goat IgG (Alexa fluor 647 conjugated, 1:500). Then, embryos were rinsed with PBS again and mounted on $0.8 \%$ LMP agarose for observation. Fluorescent images were acquired using an inverted confocal microscope (Leica SP8).

\section{Retinoic acid treatment}

To detect sensitivity to RA signaling in Wnt3a-Fz5 homozygotes, we crossed Wnt3a-Fz5 heterozygotes. RA or DMSO was injected into the abdominal cavities of female mice at E7.5, and embryos were harvested at E8.5. 


\section{Wnt vis reporter detection}

Embryos were harvested at each stage and fixed overnight in 4\% PFA. Then, they were stained with DAPI in $1 \%$ Triton X-100 solution $(1: 2,000)$ for several hours or overnight and mounted in $0.8 \%$ LMP agarose. Fluorescent images were acquired using an inverted confocal microscope (Leica SP8). Fluorescence of this reporter in the nucleus was measured in individual cells in a photon-counting mode. Epiblast cells in the region lateral to the node were individually analyzed in a single confocal plane. The area of the nuclei in each cell was identified by DAPI staining. The GFP intensity in the identified area was counted. For the cells with the top $10 \%$ GFP intensity in region II, the relative GFP intensities of the cells in contact with those highGFP cells were summarized.

\section{Quantification and statistical analysis}

Statistical analyses were performed using Excel and R software. Differences were assessed for statistical significance using T-tests. p-values $<0.05$ were considered statistically significant. Error bars in graphs indicate the standard deviation of each group.

\section{Mathematical Model}

We considered an ideal situation in which $L_{x} \times L_{y}$ epiblast cells align on a regular square lattice such that the $x$ and $y$ axes coincide with the left-right and the anterior-posterior axes (Fig. 7a). Each cell has its Wnt signaling activity as a variable $W_{i j}$, where $i$ and $j$ are the indexes of the cell on the x-y coordinate. The activity $W_{i j}$ changes in time through the production, degradation, and intercellular diffusion of Wnt molecules (Fig. 7a), and its time evolution is given by Langevin equation:

$$
\frac{d}{d t} W_{i j}=a+\alpha \frac{W_{i j}^{2}}{K^{2}+W_{i j}^{2}}-\beta W+D \sum_{k, k^{\prime} \in n . n .}\left(W_{k, k^{\prime}}-W_{i j}\right)-d_{R A}(j, t) W_{i j}+\gamma \xi_{i j}(t)
$$

The first and second terms represent the production of Wnt molecules, a constant production $\left(1^{\text {st }}\right.$ term), and autocatalytic reaction with half saturation concentration $K$ (2nd term), while the third term represents degradation with a degradation constant $\beta$. The fourth term denotes intercellular diffusion of Wnt molecules with diffusion constant $D$. The summation $\sum_{k, k^{\prime} \in \text { n.n. }}$ ( ) indicates the summation with respect to the nearest-neighbor cells around $W_{i j}$ (i.e., $W_{i \pm 1, j}$ and $\left.W_{i, j \pm 1}\right)$. RA-dependent repression is given by the fifth term. As long as the RA diffusion does not reach the region of epiblast cells $(t<t+R A)$, the value of $d_{-} R A(j, t)$ is set to 0 ; $d_{R A}(j, t)=0$. After RA diffusion reaches this region $\left(t \geq t_{R A}\right), d_{R A}(j, t)$ creates the following 
time-independent spatial gradient that increases linearly along y-axis (the anterior-posterior axis) with a slope $\Delta$ :

$$
d_{R A}(j)=\beta_{1}-\beta+j \Delta .
$$

The last term in Eq.(1) denotes the noise term: $\gamma$ and $\xi_{i j}(t)$ indicate the noise strength and independent Gaussian white noise with zero mean and variance $\left\langle\xi_{i j}(t) \xi_{i^{\prime} j^{\prime}}\left(t^{\prime}\right)\right\rangle=$ $\delta\left(t-t^{\prime}\right) \delta_{i, i} \delta_{j, j}$, respectively. Cell division events are also considered a stochastic Poisson process in the model. Each cell randomly divides at a constant rate $\lambda$. By the cell division, surrounding cells are pushed stochastically toward either the left (in the negative direction of the $\mathrm{x}$-axis with probability $1 / 4$ ), right (along the positive direction of $\mathrm{x}$-axis with probability $1 / 4$ ) or upward (along the positive direction of $y$-axis with probability $1 / 2$ ). For instance, when the left is chosen in the division of cell with $x=i$ and $y=j$, all cells with $x<i$ and $y=j$ move toward the left. The extruded cell outside the $L_{x} \times L_{y}$ lattice is removed.

Equation (1) is numerically solved by the Euler-Maruyama method with $\Delta t=10^{-5}$, while presence or absence of a cell division event is determined at each time step following the probability $\lambda L_{x} L_{y} \Delta t$. The time unit is normalized so that $\lambda=1$, while the length scale is normalized by cell length (the lattice size). The diffusion constant $D$ is set to $D=8.0$ for paracrine ( + ) cells (Fig. 7b, $\mathrm{d}$ and e) and $D=0$ for paracrine (-) cells (Fig. 7c and f). The production rate of Wnt molecules was chosen as $a=30.0$ for paracrine ( \pm ) cells (Fig. 7b, c, e and f) and $a=26.8$ for paracrine ( + )-production ( $\downarrow$ ) cells (Fig.7d). The parameter $\beta_{1}$ that represents basal degradation for $t \geq t_{R A}$ was chosen as $\beta_{1}=250.0$ for RA(-) situation (Fig.7b-d) and $\beta_{1}=252.0$ for RA(+) situation (Fig.7e and f). For other parameters, the following values were used: $L_{x}=L_{y}=50, \alpha=522.0, K=1.12, \beta=220.0, \Delta=0.2, \gamma=3.0$.

\section{Acknowledgements}

We thank the Spectrography and Bioimaging Facility of the NIBB Core Research Facilities for their technical support. We also thank Drs. Takahashi and Mizuno at the University of Tsukuba for generating Wnt3a-Fzd5 knock-in mice using CRISPR/Cas9-mediated genome editing and Dr. Fujimori at NIBB for providing mice and technical support. Dr. Aoki at NIBB and ExCELLS and all members of S.T.'s laboratory are gratefully acknowledged for helpful discussions. 


\section{Author contributions}

Y.H. performed the majority of experiments, participated in their planning, and wrote the text.

N.S. formulated the mathematical concept, conducted computer modeling, and wrote the text.

Y.M. participated in the planning of the experiments and discussion of the results. T.S.

participated in generation of mouse mutants. T.T. generated Wnt reporter mice. H.N. participate

in modeling. S.T. formulated the initial key hypothesis, organized all the work, planned

experiments, and wrote the text. All authors reviewed and approved the manuscript.

\section{Funding}

This work was supported by the following programs: Grants-in-Aid for Scientific Research (B), 18H02454 and 21H02498 to ST, Grants-in-aid for Scientific Research on Innovative Areas, 24111002, 17H05782, 19H04797 to ST, from the Japan Society for the Promotion of Science. Additional support came from grants from National Institutes of Natural Sciences (NINS Joint Research Program to ST) and the Cooperative Study Program of Exploratory Research Center on Life and Living Systems (ExCELLS; program Nos. 21-102 to HN and NS).

\section{Competing financial interests}

The authors declare no competing or financial interests. 


\section{References}

1. Stern, C. D. et al. Head-tail patterning of the vertebrate embryo: One, two or many unresolved problems? International Journal of Developmental Biology 50, 3-15 (2006).

2. Tzouanacou, E., Wegener, A., Wymeersch, F. J., Wilson, V. \& Nicolas, J. F. Redefining the Progression of Lineage Segregations during Mammalian Embryogenesis by Clonal Analysis. Developmental Cell 17, 365-376 (2009).

3. Cambray, N. \& Wilson, V. Two distinct sources for a population of maturing axial progenitors. Development 134, 2829-2840 (2007).

4. Cambray, N. \& Wilson, V. Axial progenitors with extensive potency are localised to the mouse chordoneural hinge. Development 129, 4855-4866 (2002).

5. Tam, P. P. \& Beddington, R. S. The formation of mesodermal tissues in the mouse embryo during gastrulation and early organogenesis. Development (Cambridge, England) 99, 109-26 (1987).

6. Wilson, V., Olivera-Martinez, I. \& Storey, K. G. Stem cells signals and vertebrate body axis extension. Development vol. 136 1591-1604 (2009).

7. Wilson, V. \& Beddington, R. S. Cell fate and morphogenetic movement in the late mouse primitive streak. Mechanisms of development 55, 79-89 (1996).

8. Takada, S. et al. Wnt-3a regulates somite and tailbud formation in the mouse embryo. Genes \& development 8, 174-89 (1994).

9. Cunningham, T. J., Kumar, S., Yamaguchi, T. P. \& Duester, G. Wnt8a and Wnt3a cooperate in the axial stem cell niche to promote mammalian body axis extension. Developmental dynamics : an official publication of the American Association of Anatomists 244, 797-807 (2015).

10. Zhao, X. \& Duester, G. Effect of retinoic acid signaling on Wnt/ $\beta$-catenin and FGF signaling during body axis extension. Gene Expression Patterns 9, 430435 (2009).

11. Liu, P. et al. Requirement for Wnt3 in vertebrate axis formation. Nature genetics 22, 361-5 (1999).

12. Tenin, G. et al. The chick somitogenesis oscillator is arrested before all paraxial mesoderm is segmented into somites. BMC Developmental Biology 10 , (2010).

13. Rivera-Pérez, J. A. \& Magnuson, T. Primitive streak formation in mice is preceded by localized activation of Brachyury and Wnt3. Developmental Biology 288, 363-371 (2005). 
14. Yamaguchi, T. P., Takada, S., Yoshikawa, Y., Wu, N. \& McMahon, A. P. T (Brachyury) is a direct target of Wnt3a during paraxial mesoderm specification. Genes \& development 13, 3185-90 (1999).

15. Anderson, M. J. et al. TCreERT2, a Transgenic Mouse Line for Temporal Control of Cre-Mediated Recombination in Lineages Emerging from the Primitive Streak or Tail Bud. PLOS ONE 8, (2013).

16. Garriock, R. J. et al. Lineage tracing of neuromesodermal progenitors reveals novel wnt-dependent roles in trunk progenitor cell maintenance and differentiation. Development 142, 1628-1638 (2015).

17. Imuta, Y., Kiyonari, H., Jang, C. W., Behringer, R. R. \& Sasaki, H. Generation of knock-in mice that express nuclear enhanced green fluorescent protein and tamoxifen-inducible Cre recombinase in the notochord from Foxa2 and T loci. Genesis 51, 210-218 (2013).

18. Koch, F. et al. Antagonistic Activities of Sox2 and Brachyury Control the Fate Choice of Neuro-Mesodermal Progenitors. Developmental Cel/ 42, 514-526.e 7 (2017).

19. Yoshikawa, Y., Fujimori, T., McMahon, A. P. \& Takada, S. Evidence that absence of Wnt-3a signaling promotes neuralization instead of paraxial mesoderm development in the mouse. Developmental biology 183, 234-42 (1997).

20. Dunty, W. C. et al. Wnt3a/ $\beta$-catenin signaling controls posterior body development by coordinating mesoderm formation and segmentation. Development 135, 85-94 (2008).

21. Dunty, W. C., Kennedy, M. W. L., Chalamalasetty, R. B., Campbell, K. \& Yamaguchi, T. P. Transcriptional profiling of Wnt3a mutants identifies Sp transcription factors as essential effectors of the Wnt/ $\beta$-catenin pathway in neuromesodermal stem cells. PLoS ONE 9, e87018 (2014).

22. Galceran, J., Hsu, S. C. \& Grosschedl, R. Rescue of a Wht mutation by an activated form of LEF-1: regulation of maintenance but not initiation of Brachyury expression. Proceedings of the National Academy of Sciences of the United States of America 98, 8668-73 (2001).

23. Martin, B. L. \& Kimelman, D. Regulation of Canonical Wnt Signaling by Brachyury Is Essential for Posterior Mesoderm Formation. Developmental Cell 15, 121-133 (2008).

24. Martin, B. L. \& Kimelman, D. Brachyury establishes the embryonic mesodermal progenitor niche. Genes and Development 24, 2778-2783 (2010). 
25. Shinozuka, T., Takada, R., Yoshida, S., Yonemura, S. \& Takada, S. Wnt produced by stretched roof-plate cells is required for the promotion of cell proliferation around the central canal of the spinal cord. Development 146, (2019)

26. Greco, T. L. et al. Analysis of the vestigial tail mutation demonstrates that Wnt-3a gene dosage regulates mouse axial development. Genes \& development 10, 313-24 (1996).

27. Tsakiridis, A. et al. Distinct Wnt-driven primitive streak-like populations reflect in vivo lineage precursors. Development 141, 1209-1221 (2014).

28. Takemoto, T. et al. R26-WntVis reporter mice showing graded response to Wnt signal levels. Genes to Cells 21, 661-669 (2016).

29. Yamaguchi, T. P. Genetics of Wnt signaling during early mammalian development. Methods in Molecular Biology 468, 287-305 (2008).

30. Abu-Abed, S. et al. The retinoic acid-metabolizing enzyme, CYP26A1, is essential for normal hindbrain patterning, vertebral identity, and development of posterior structures. Genes \& development 15, 226-40 (2001).

31. Briscoe, J. \& Small, S. Morphogen rules: Design principles of gradientmediated embryo patterning. Development (Cambridge) vol. 142 3996-4009 (2015).

32. Gurdon, J. B. \& Bourillot, P. Y. Morphogen gradient interpretation. Nature 413, 797-803 (2001).

33. Christian, J. L. \& Moon, R. T. Interactions between Xwnt-8 and Spemann organizer signaling pathways generate dorsoventral pattern in the embryonic mesoderm of Xenopus. Genes and development 7, 13-28 (1993).

34. Petersen, C. P. \& Reddien, P. W. Wnt Signaling and the Polarity of the Primary Body Axis. Cell vol. 139 1056-1068 (2009).

35. Shimizu, T., Bae, Y. K., Muraoka, O. \& Hibi, M. Interaction of Wnt and caudalrelated genes in zebrafish posterior body formation. Developmental Biology 279, 125-141 (2005).

36. Mitrophanov, A. Y. \& Groisman, E. A. Positive feedback in cellular control systems. BioEssays : news and reviews in molecular, cellular and developmental biology 30, 542-55 (2008).

37. Abu-Abed, S. et al. Developing with lethal RA levels: Genetic ablation of Rarg can restore the viability of mice lacking Cyp26a1. Development vol. $1301449-$ 1459 (2003). 
38. Niederreither, K., McCaffery, P., Dräger, U. C., Chambon, P. \& Dollé, P. Restricted expression and retinoic acid-induced downregulation of the retinaldehyde dehydrogenase type 2 (RALDH-2) gene during mouse development. Mechanisms of development 62, 67-78 (1997).

39. MacLean, G. et al. Cloning of a novel retinoic-acid metabolizing cytochrome P450, Cyp26B1, and comparative expression analysis with Cyp26A1 during early murine development. Mechanisms of development 107, 195-201 (2001).

40. Sakai, Y. et al. The retinoic acid-inactivating enzyme CYP26 is essential for establishing an uneven distribution of retinoic acid along the anterio-posterior axis within the mouse embryo. Genes \& development 15, 213-25 (2001).

41. Gurdon, J. B. A community effect in animal development. Nature 336, 772-4 (1988).

42. Gurdon, J. B., Lemaire, P. \& Kato, K. Community effects and related phenomena in development. Cel/ 75, 831-4 (1993).

43. Saka, Y., Lhoussaine, C., Kuttler, C., Ullner, E. \& Thiel, M. Theoretical basis of the community effect in development. BMC Systems Biology 5, (2011).

44. Tsukiyama, T. et al. Molecular Role of RNF43 in Canonical and Noncanonical Wnt Signaling. Molecular and Cellular Biology 35, 2007-2023 (2015).

45. Laemmli, U. K. Cleavage of structural proteins during the assembly of the head of bacteriophage T4. Nature 227, 680-5 (1970).

46. Roelink, H. \& Nusse, R. Expression of two members of the Wnt family during mouse development--restricted temporal and spatial patterns in the developing neural tube. Genes \& development 5, 381-8 (1991).

47. Mansouri, A. et al. Paired-related murine homeobox gene expressed in the developing sclerotome, kidney, and nervous system. Developmental dynamics: an official publication of the American Association of Anatomists 210, 53-65 (1997).

48. Liu, C., Wang, Y., Smallwood, P. M. \& Nathans, J. An essential role for Frizzled5 in neuronal survival in the parafascicular nucleus of the thalamus. Journal of Neuroscience 28, 5641-5653 (2008). 


\section{Figure Legends}

Fig. 1. In vitro activity of WNT3A-FZD5 and generation of Wnt3a-Fzd5 knock-in mice (a) Schematic fig. of WNT3A-FZD5 protein, comparing it with WNT3A and GFP-WNT3A. (b, c) Wnt signaling activity of each construct shown in A. Wnt signaling activity was monitored in HEK293T (STF293) cells stably expressing the SuperTOPFLASH reporter. In (b), Wnt activity was monitored at 24, 48, and $72 \mathrm{~h}$ after transfection of each plasmid into STF293 cells. In (c), paracrine Wnt activity was monitored in co-cultures of Wnt-expressing HEK293T cells with STF293 cells at 24 and $48 \mathrm{~h}$ after transfection. Differences were assessed for statistical significance using a T-test; *** $\mathrm{P}<0.001 ; * * \mathrm{P}<0.01 ; * \mathrm{P}<0.05 ; \mathrm{P}>0.05$; NS (not statistically significant). Error bars in the graph indicate the standard deviation of each group. (d-g) Expression of mouse Wnt $3 a$ and human $F z d 5$ in $W n t 3 a-F z d 5$ heterozygous ( $W n t 3 a^{+/ F z d 5}$ ) embryos. Whole-mount in situ hybridization was carried out using probes of mouse Wnt3a (d, e) or human $F z d 5$ (f, g) in wild type (d, f) and $W n t 3 a^{+/ F z d 5}$ (e, g) embryos at E10.5. Images are highlighted on the tailbud (d, e, f, g) and the roof plate of neural tube (d', e', f', g'). Numbers of stained embryos are indicated by " $n="$ in the images. (h) Western blotting analysis of proteins prepared from E8.5 embryos with anti-mouse Wnt3a antibody. Samples prepared from two embryos were applied to each lane. Note that bands with the predicted molecular weight of WNT3A-FZD5 were detected both in $W n t 3 a-F z d 5$ heterozygous $(+/ F z d 5)$ and homozygous (Fzd5/Fzd5) embryos. (i-k) Sagittal views of wt (i), $W n t 3 a^{+/ F z 5}$ (j) and $W n t 3 a^{F z 5 / F z 5}$ (k) embryos at E10.5. i', j', and k' are magnified images of i, j, and k, respectively. i', j', and k" are drawings of the images of i', j', and k', respectively. (1-n) Transverse sections of the neural tube of $W T\left(1,1\right.$ ', 1''), Wnt $3 a^{+/ F z 5}$ (m, m', m'') and $W n t 3 a^{F z 5 / F z 5}$ (n, n', n'') embryos at E11.5. Sections at the forelimb $(1, m, n)$, the intermediate between fore and hindlimb (l', m', n') and the hindlimb (l', m', n'”) levels are shown. Scale bars: 1 mm (d-g', i-k'), $100 \mu \mathrm{m}$ (1-n'), $200 \mu \mathrm{m}$ (n'). HL: Hindlimb. FL: forelimb.

\section{Fig. 2. Expression of mesoderm and neural marker genes in Wnt3a-Fzd5 homozygous embryos}

(a-v) Expression of mesoderm and neural marker genes in embryos at E10.5. Whole-mount in situ hybridization was carried out using probes of Bra (a-f), Tbx6 (g-1), Uncx4.1 (m-q), and Sox2 (r-v) in wt \& $W n t 3 a^{+/ F z 5}(\mathrm{a}, \mathrm{g}, \mathrm{m}, \mathrm{r}), W n t 3 a^{F z 5 / F z 5}(\mathrm{~b}, \mathrm{~h}, \mathrm{n}, \mathrm{s}), W n t 3 a^{+/ v t}(\mathrm{c}, \mathrm{i}, \mathrm{o}, \mathrm{t}), W n t 3 a^{v t /-}(\mathrm{d}, \mathrm{j}, \mathrm{p}$, $\mathrm{u}), W n t 3 a^{+-}(\mathrm{e}, \mathrm{k}, \mathrm{q}, \mathrm{v})$ and $W n t 3 a^{--}(\mathrm{f}, 1)$ embryos at E10.5. Red dotted lines indicate tail regions. (w-ad) Expression of mesoderm and neural marker genes in embryos at E12.5. Whole-mount in situ hybridization was carried out using probes of $B r a(\mathrm{w}, \mathrm{x}, \mathrm{y})$, and $\operatorname{Tbx} 6(\mathrm{z}, \mathrm{aa}, \mathrm{ab})$ in $W T$ \& $W n t 3 a^{+/ F z 5}(\mathrm{w}, \mathrm{z}), W n t 3 a^{F z 5 / F z 5}$ (x, y, aa, ab) embryos at E12.5. Tail regions of stained embryos were cut out and shown in $y$ and $a b . x^{\prime}$ and aa are drawings of the images of $x$ and aa 
respectively. Numbers of stained embryos are indicated by " $n="$ in the images. Scale bars: 1 mm. HL: Hindlimb.

Fig. 3. Differentiation of Wnt-positive progenitor cells in the neural tube and somites in Wnt3a-Fzd5 homozygous embryos.

(a) Experimental procedure. Cells once activated by Wnt signaling, which is monitored by Axin2-CreERT2 expression, were eternally labelled by expression of tdTomato. Tamoxifen (TM) was injected into pregnant females at $8.5 \mathrm{dpc}$ and embryos were fixed at E10.5. (b, c) Distribution of tdTomato-labelled cells at the posterior hindlimb level in $W n t 3 a^{+/ F z d 5}$ (b) and $W n t 3 a^{F z d 5 / F z d 5}$ (c) embryos at E10.5. Merged images with DAPI-staining are also indicated (b', c'). Neural tube and dermomyotome, which is derived from somite, are outlined with white and orange dotted lines, respectively. Squares framed by green dotted lines indicate the area around the nephric duct. The percentage of tdTomato-positive cells (d, e) and total cell number (f, g) in somite ( $\mathrm{d}, \mathrm{f})$, nephric duct (e) and neural tube ( $\mathrm{g}$ ) at the posterior hindlimb level in Wnt $3 a^{+/ F z d 5}$ and $W n t 3 a^{F z d 5 / F z d 5}$ embryos at E10.5. Numbers or percentages of labelled cells (mean \pm s.d.) per section are shown. Differences were assessed for statistical significance using a T-test; ***, P < 0.001 ; **, $\mathrm{P}<0.01$; NS, not statistically significant $(\mathrm{P}>0.05)$. Error bars in the graph mean the standard deviation of each group. Scale bars: $100 \mu \mathrm{m}$

\section{Fig. 4. NMP cells in Wnt3a-Fzd5 homozygous embryos}

(a-1) Whole-mount staining of Wnt3a-Fzd5 heterozygous (a, c, e, g, j, 1, n) and homozygous (b, $\mathrm{d}, \mathrm{f}, \mathrm{h}, \mathrm{k}, \mathrm{m}, \mathrm{o}$ ) embryos at E8.75. Maximum intensity projection images of posterior ends of embryos stained with anti-SOX2 (magenta) and anti-BRA (green) antibodies are shown in A and B. To quantify the number of SOX2/BRA double-positive cells, single-plane images of medial (I) and lateral (II) regions lateral to the node were analyzed (c-p). Images of DAPI staining (blue; c, d, j, k), and merged images of staining with anti-SOX2 (magenta) and antiBRA (green) antibodies (e, f, l, m) are shown. Summarized schematic fig.s (g, h, n, o) and diagrams (i, p) are also shown. The size of the medial and lateral regions is $50 \mu \mathrm{m} \times 100 \mu \mathrm{m}$. Two embryos were examined for each genotype. (q-x) Whole-mount staining of $W n t 3 a^{+/ F z d 5}(\mathrm{q}$, $\mathrm{u}), W n t 3 a^{F z d 5 / F z d 5}(\mathrm{r}, \mathrm{v}), W n t 3 a^{+/ v t}(\mathrm{~s}, \mathrm{w})$, and $W n t 3 a^{v t /}(\mathrm{t}, \mathrm{x})$ embryos at E11.5. Maximum intensity projection images of posterior ends of embryos stained with DAPI (blue; q-t), and with antiSOX2 (magenta) and anti-BRA (green) antibodies (q'-t'), are shown. Single-plane images of the areas indicated with yellow-lined boxes in q'-t' are magnified in $\mathrm{u}-\mathrm{x}$, respectively. Images of staining with anti-SOX2 (magenta; u-x) and anti-BRA (green; u'-x') antibodies, as well as merged images ( $u$ "-x") are shown. The yellow-lined box is a square with one side $=100 \mu \mathrm{m}$. Arrowheads in v" indicate a small number of Sox $2 /$ Bra-positive cells. Note that there are no 
SOX2 and BRA double-positive cells in $W n t 3 a^{v t /-}(\mathrm{t}, \mathrm{x})$. The number of stained embryos is indicated by "n=". Scale bars: $100 \mu \mathrm{m}(\mathrm{a}, \mathrm{b}, \mathrm{q}-\mathrm{t})$

Fig. 5. Wnt signaling in the epibrast cell population of Wnt3a-Fzd5 homozygous embryos Wnt signaling activity in individual epiblast cells was visualized using mouse embryos carrying an EGFP-reporter gene, expression of which is specifically activated by Wnt signaling. The observation scheme of embryos at cylinder stage(E7.0 and E7.5 : a) and post-somitegenesis stage(E8.5 and E8.75 : f) . Eye marks in $(a, f)$ indicate the direction of observation. Blue boxes in (f) indicate somites. Wnt signaling activity was monitored in $W n t 3 a-F z d 5$ heterozygous (b, d, g, k, q) and homozygous (c, e, h, 1, r) embryos at E7.0 (b, c), E7.5 (d, e), E8.0 (g, h), E8.75 (k, 1), and E9.5(q, r). Wnt signaling activity was also visualized in $+/ v t(\mathrm{i}, \mathrm{m}, \mathrm{s})$ and $v t /-(\mathrm{j}, \mathrm{n}, \mathrm{t})$ embryos at E8.0 (i, j), E8.75 (m, n), and E9.5(s, t). Each embryos, magnified images of the areas indicated by boxes. Note that Wnt signaling activity is not obviously changed in Wnt3a-Fzd5 homozygous embryos at E7.0 (b, c) or at E7.5 (d, e). In E8.0 and E8.75 embryos, magnified images of the CLE in (f) are shown in each genotyped embryo. (Areas $=100 \times 100 \mathrm{~mm}$.) The magnified images were taken at a single confocal plane while the others were processed by maximum intensity projection. GFP intensity in individual cells in CLE was quantified in each genotyped embryo at E8.0 (o) and E8.75 (p). Two embryos were examined for each genotype. Box plots indicate the first and third quartiles and the median. Scale bars: $100 \mu \mathrm{m}$. The star in the E9.5 shows the nephric duct.

Fig. 6. Effect of retinoic acid on the epiblast cell population of Wnt3a-Fzd5 homozygous embryos

(a-g) Analysis of retinoic acid (RA)-treated embryos at E8.75. Experimental schemes are shown in ( $a, b)$. Dorsal images of DMSO (c, d) or10 mM RA (e, f) treated Wnt $3 a-F z d 5$ heterozygous (c, e) and homozygous (d, f) embryos at E8.75 stained with DAPI (blue). Results of quantification of the width at NSB (g) and the length posterior to NSB (h) in each genotyped embryo are shown. Note that RA treatment enhances the abnormality in gross morphology specifically in $W n t 3 a-F z d 5$ homozygous embryos. Red arrows indicate the width at NSB while orange arrows indicate the length posterior to NSB. Differences were assessed for statistical significance using a T-test; *** $\mathrm{P}<0.001 ; * * \mathrm{P}<0.01 ; * \mathrm{P}<0.05 ; \mathrm{P}>0.05$; n.s. (not statistically significant). Error bars in the graph indicate the standard deviation of each group. Scale bars: $100 \mu \mathrm{m}$. (i-m) Analysis of retinoic acid (RA)-treated embryos at E8.5. These embryos were treated with RA 7.5 days post coitum. Wnt signaling activity in individual epiblast cells was visualized as shown in Fig. 5(f). Dorsal images, processed by maximum intensity projection of DMSO- (i, k) or $10 \mathrm{mM} \mathrm{RA}-(\mathrm{j}, 1)$ treated $W n t 3 a-F z d 5$ heterozygous $(\mathrm{i}, \mathrm{j})$ 
and homozygous $(\mathrm{k}, 1)$ embryos at E8.5 are shown. Images of a single confocal plane in CLE . The size of these areas is $100 \mu \mathrm{m} \times 100 \mu \mathrm{m}$ and their positions in the epiblast are identical to those shown in Fig. 5(f). GFP intensity in individual cells in CLE is summarized in (m). Two embryos were examined for each genotype. Box plots indicate the first and third quartiles and the median. Differences were assessed for statistical significance using a wilcoxon signed-rank test; ${ }^{* *} \mathrm{P}<0.001 ; * * \mathrm{P}<0.01 ; * \mathrm{P}<0.05 ; \mathrm{P}>0.05$; n.s. (not statistically significant). In (a) and (h), PS indicates the primitive streak and blue boxes indicate somites. Scale bars: $100 \mu \mathrm{m}$.

Fig. 7. Mathematical Model for Examining the Effect of Intercellular Communication in Cell Populations

(a) Schematic diagram showing parameters used in the model. We assumed a virtual space corresponding to the cell sheet of the epiblast. This virtual space is divided into 50 X 50 sections along the antero-posterior and medio-lateral axes. Each section corresponds to a single cell in the epiblast. Wnt activity (W) is determined by parameters such as the rate of production and degradation of Wnt protein, the rate of amplification or reduction by positive feedback, the rate of intercellular exchange of Wnt protein, the rate of inhibition by RA, and fluctuating noise that affects Wnt activity. It is assumed that cell division occurs randomly and that dividing daughter cells are extruded in one section in either the left, right, or anterior direction in a 1:1:2 ratio. (b-f) Spatial patterns of Wnt activity in a virtual sheet of cells. Examples of the spatial pattern in the presence $(b, d, e)$ or absence $(c, f)$ of the paracrine function of Wnt are shown at the same time point (mean division time $\mathrm{t}=3.00$ ) after addition of $\mathrm{RA}(\mathrm{t}=0)$. In the condition of $\mathrm{D}$, the Wnt production rate is reduced (see Method). The spatial patterns of Wnt activity were calculated in the absence (b-d) and presence (e, f) of uniformly supplied RA. (g) Time course of Wnt-positive cells in a virtual sheet of cells. The time course of the proportion of Wnt-positive cells ( $>50 \%$ of maximum activity) at the same spatial level ( $y=35$ in $b$-d) along the anterior-posterior axis in a virtual sheet of cells is shown. Orange and blue lines indicate the result with and without the paracrine function of Wnt, respectively. A green line indicates the result obtained in the condition where the Wnt production rate is reduced in the presence of the paracrine function of Wnt. Solid and dashed lines indicate results obtained in the absence and presence of uniformly supplied RA, respectively. (h) Schematic representation showing the effect of Wnt paracrine in the epiblast cell population. Prior to somite formation (E7.0-E8.0), Wnt activity in each epiblast cell is dramatically increased by positive feedback regulation mediated by Wnt3a and Bra. During this period, no obvious difference was observed between control and embryos lacking paracrine Wnt signaling (Wnt3a-Fzd5 
homozygous embryos). After the onset of somite formation (after E8.0), the number of Wnt weak cells was increased by the antagonistic effect of RA, which is synthesized in somites, but a small number of Wnt-strong cells remain for a long period in embryos lacking paracrine Wnt signaling (paracrine (-)). This increased heterogeneity in Wnt signaling is compensated for by intercellular exchange of Wnt ligands between epiblast cells (paracrine $(+))$.

\section{Extended Data Fig. 1. WNT3A-FZD5 was not detected in culture supernatant.}

Western blotting analysis of cell lysate (a) and culture supernatant (b) prepared from WNT3A and WNT3A-FZD5 expressing HEK293T cells at 24, 48, and $72 \mathrm{~h}$ after transfection. While the expression level of WNT3A-FZD5 was similar to WNT3A in the cell lysate, WNT3A-FZD5 was not detectable in culture supernatant. Red and blue arrowheads indicate bands corresponding to the predicted molecular weights of WNT3A-FZD5 and WNT3A, respectively.

\section{Extended Data Fig. 2. Generation of Wnt3a-Fz5 knock-in mice.}

(a-f) Generation the Wnt3a-Fzf5 knock-in allele. A schematic fig. indicates the mouse Wnt3a locus and the Wnt3a-Fzd5 knock-in allele is shown in (a). In the knock-in allele, human Frizzled5 (blue) fused with 2 myc tags (green) is inserted at the C-terminus of mouse Wnt3a. The knock-in event was confirmed by PCR analysis using the primer sets indicated in $b$. The results of PCR analyses are shown (c-f). Prime sets are indicated on the upper side of each fig.. Band sizes indicated by colored arrowheads correspond to the predicted sizes shown in b. (g-j) Whole image of wt $(\mathrm{g}, \mathrm{i})$ and $W n t 3 a^{+/ F z d 5}(\mathrm{~h}, \mathrm{j})$ embryos at E10.5 hybridized with $W n t 3 a(\mathrm{~g}, \mathrm{~h})$ or $h F z d 5(\mathrm{i}, \mathrm{j})$ probes. Magnified images of posterior bodies and dorsal views of these embryos are shown in Fig. 1d-g. Scale bars: $1 \mathrm{~mm}$. (k) The proportion of individuals of each genotype during embryonic development and immediately after birth.

\section{Extended Data Fig. 3. The source of ventral neural cells loses Wnt signaling after E7.5 during development of Wnt3a-Fzd5 heterozygous embryos.}

(a) Experimental procedure. Cells once activated by Wnt signaling, which is monitored by Axin2-CreERT2 expression, were eternally labelled by expression of tdTomato. Tamoxifen (TM) was injected to pregnant females at $7.5 \mathrm{dpc}(\mathrm{b}, \mathrm{d})$ or $8.5 \mathrm{dpc}(\mathrm{c}, \mathrm{e})$ and embryos were fixed at E10.5. Whole-mount bright field images (b, c) and tdTomato staining are also indicated (b', c'). Distribution of tdTomato-labelled cells at the posterior hindlimb level in $W n t 3 a^{+/ F z d 5}$ embryos at E10.5(d, e). Scale bars: $100 \mu \mathrm{m}$ 
Extended Data Fig. 4. Synergistic effect of Wnt3a-Frizzled and Bra on the posterior development of body axis elongation

Wnt $3 a^{+/ F z d 5} ; \mathrm{Bra}^{+/+}(\mathrm{a}), \mathrm{Wnt}_{3} \mathrm{a}^{+/+} ; \mathrm{Bra}^{+-}$(b) and $\mathrm{Wnt}_{3 \mathrm{a}^{+/ F z d 5}} ; \mathrm{Bra}^{+/}$(c) embryos stained by wholemount in situ hybridization using the Sox2 probe are shown. Embryos were fixed at E11.5. a', b', and c' are magnified images of a, b, and c, respectively. Note that Wnt $3 a-F z d 5$ and Bra compound heterozygous embryos $\left(\mathrm{Wnt}_{3} \mathrm{a}^{+/ \mathrm{Fz} d 5} ; \mathrm{Bra}^{+/}(\mathrm{c})\right)$ impair the posterior development of body axis elongation while embryos heterozygous for either of them appear normal. Scale bars: $1 \mathrm{~mm}$.

\section{Extended Data Fig. 5. Characteristics of $\mathrm{Wnt}_{3} \mathrm{a}^{\mathrm{vt} / \text { - }}$ embryos}

(a-c) Sagittal views of wt (a), Wnt3 $a^{+/ v t}$ (b) and $W n t 3 a^{v t /}$ (c) embryos at the E11.5. a', b', and c' are magnified images of $a, b$, and c, respectively. a', b", and c", are drawings of the images of a', b', and c', respectively. (d-f) Wnt3a expression was detected by whole-mount in situ hybridization of wt (d), Wnt $3 a^{+/ v t}$ (e) and $W n t 3 a^{v t /-}$ (f) embryos at E9.5. Dorsal views of the posterior region of each embryo are indicated. Red dotted lines indicate the outer edge of the tail. In Wnt $3 a^{v t /}$ embryos, Wnt3a expression is highly decreased at this stage. (g, h) Wholemount in situ hybridization of $W n t 3 a^{+/ v t}$ and $W n t 3 a^{v t /-}$ embryos at E12.5 using $B r a(\mathrm{~g})$ and Tbx6(h) probes. In contrast to Wnt3a-Fzd5 homozygous embryos, the expression of Bra nad Tbx6 is not detectable in $W n t 3 a^{v t /-}$ embryos. Scale bars: $1 \mathrm{~mm}$.

Extended Data Fig. 6. The phenotype of Wnt3a-Fzd5 homozygous embryos can be rescued, depending on intercellular signaling of Wnt3a

(a-d) Sagittal views of $W n t 3 a^{+/ F z 5}$ (a, a', a'), Wnt $3 a^{F z 5 / F z 5}$ (b, b', b' '), Wnt $3 a^{F z 5 /-}\left(\mathrm{c}, \mathrm{c}^{\prime}, \mathrm{c}^{\prime \prime}\right)$ and $W n t 3 a^{v t / F z 5}\left(\mathrm{~d}, \mathrm{~d}^{\prime}, \mathrm{d}^{\prime}\right.$ ') embryos at the E10.5. a', b', c', and d' are magnified images of a, b, c, and d, respectively. a', b', c', and d"' are drawings of the images of a', b', c', and d', respectively. (e-h) Whole-mount in situ hybridization of $W n t 3 a^{+/ F z 5}$ (e), $W n t 3 a^{F z 5 / F z 5}$ (f), $W n t 3 a^{F z 5 /}(\mathrm{g})$ and $W n t 3 a^{v t / F z 5}(\mathrm{~h})$ embryos at E10.5 with Bra probe. Red dotted lines indicate the edge of the body posterior to the hindlimb. Scale bars: $1 \mathrm{~mm}$.

\section{Extended Data Fig. 7. Summary plots of Bra and Sox2 signal intensities examined by} immunohistochemistry.

(a) Schematic fig. showing the area examined. (b) Summary plots of Bra and Sox2 signal intensities in medial (I) and lateral (II) areas at the node-streak border in Wnt3a-Fzd5 heterozygous and homozygous embryos. Two embryos were examined for each genotype. Measurements for each cell are plotted according to levels of Bra (x-axis) and Sox (y-axis). Levels of Bra and Sox 2 in each cell were normalized by the average of levels of Bra and Sox 2 
level in the medial area of Wnt3a-Fzd5 heterozygous embryos. Cells located between the two dashed lines were defined as Bra and Sox2 double-positive cells.

\section{Extended Data Fig. 8. Wnt signaling in the epiblast cell population of Wnt3a knock-out embryos}

Wnt signaling activity in individual epiblast cells was visualized using mouse embryos carrying an EGFP-reporter gene, expression of which is specifically activated by Wnt signaling. Wnt signaling activity was monitored in Wnt3a knock-out (b, d) and WT (a, c) embryos at E7.5 (a, b) and E8.5 (c, d). Note that Wnt signaling is drastically reduced at E7.5 (e) and completely lost at E8.5 (f) in Wnt3a null embryos, suggesting that Wnt activity at and after E8.5 epiblast is dependent on only Wnt3a ligand. Scale bars: $100 \mu \mathrm{m}$.

\section{Extended Data Fig. 9. Temporal changes of the spatial profile of Wnt-positive cells as} simulated by our mathematical model.

Spatial profiles of the proportion of Wnt-positive cells ( $>50 \%$ of maximum activity) in the virtual space are indicated at $0(\mathrm{~T}=\mathrm{TR}), 0.2(\mathrm{~T}=\mathrm{TR}+0.2), 1(\mathrm{~T}=\mathrm{TR}+1), 2(\mathrm{~T}=\mathrm{TR}+2), 3$ $(\mathrm{T}=\mathrm{TR}+3)$ mean division time after addition of RA. Spatial profiles under combined conditions with and without Wnt-mediated intercellular communication and with and without uniformly supplied RA are shown. Also shown is the spatial profile under the condition of Wnt-mediated intercellular communication and a reduced rate of Wnt production. In Fig. 7G, the time course of the proportion of Wnt-positive cells at the same spatial level $(y=35)$ is summarized in a single graph. A and $\mathrm{P}$ indicate anterior and posterior, respectively.

\section{Extended Data Movie 1 Simulation of the time course of the spatial pattern of Wnt} signaling activity in a hypothetical epiblast using our mathematical model.

(a) Time course of Wnt activity in the hypothetical epiblast in the presence of intercellular exchange of Wnt ligands.

(b) Time course of Wnt activity in the hypothetical epiblast in the absence of intercellular exchange of Wnt ligands.

(c) Time course of Wnt activity in the hypothetical epiblast in the presence of intercellular exchange of Wnt ligands, but reduced Wnt production.

(d) Time course of Wnt activity in the hypothetical epiblast with uniform addition of RA in the presence of intercellular exchange of Wnt ligands.

(e) Time course of Wnt activity in the hypothetical epiblast with uniform addition of RA in the absence of intercellular exchange of Wnt ligands. 
a

WNT3A (39.2 kDa)

mouse WNT3A

GFP-WNT3A (67.7 kDa)

\begin{tabular}{|l|l|l|}
\hline EGFP & mouse WNT3A \\
\hline
\end{tabular}

WNT3A-FZD5 (104.2 kDa)

mouse WNT3A III hFZD5
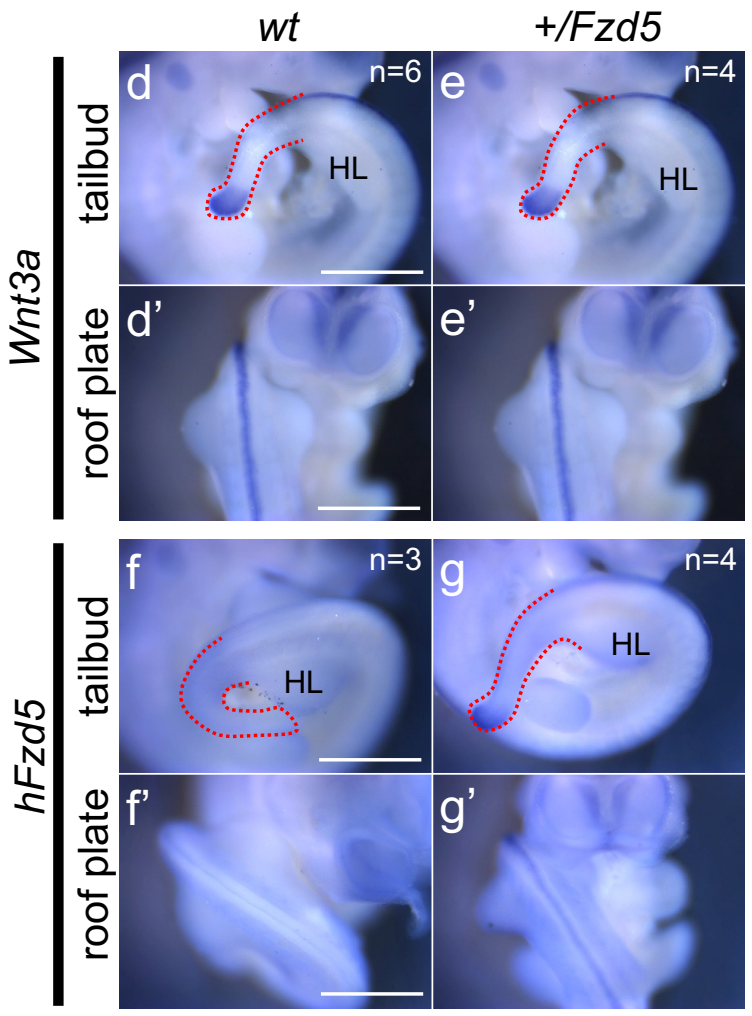

h

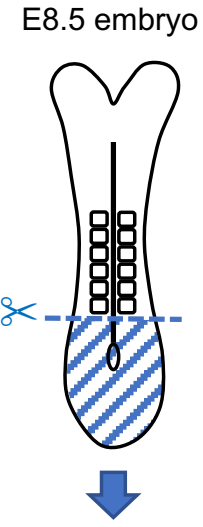

Western analysis b

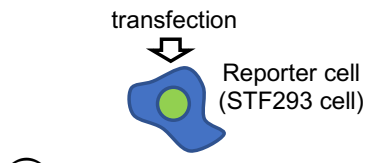

ร்

त्่ 3.0

2.0

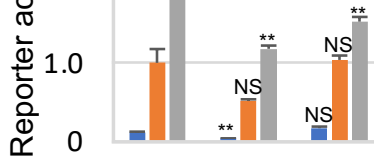

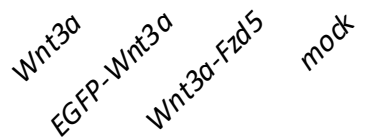

- $24 \square 4 \quad 72$

hours after transfection
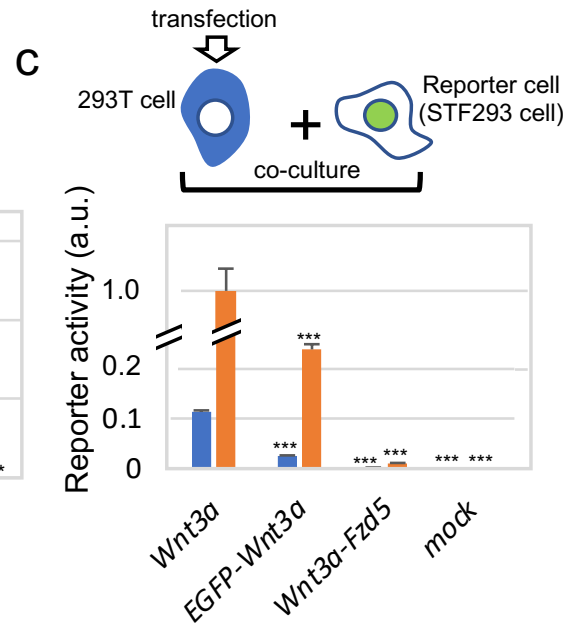

$-24 \square 48$

hours after co-culture
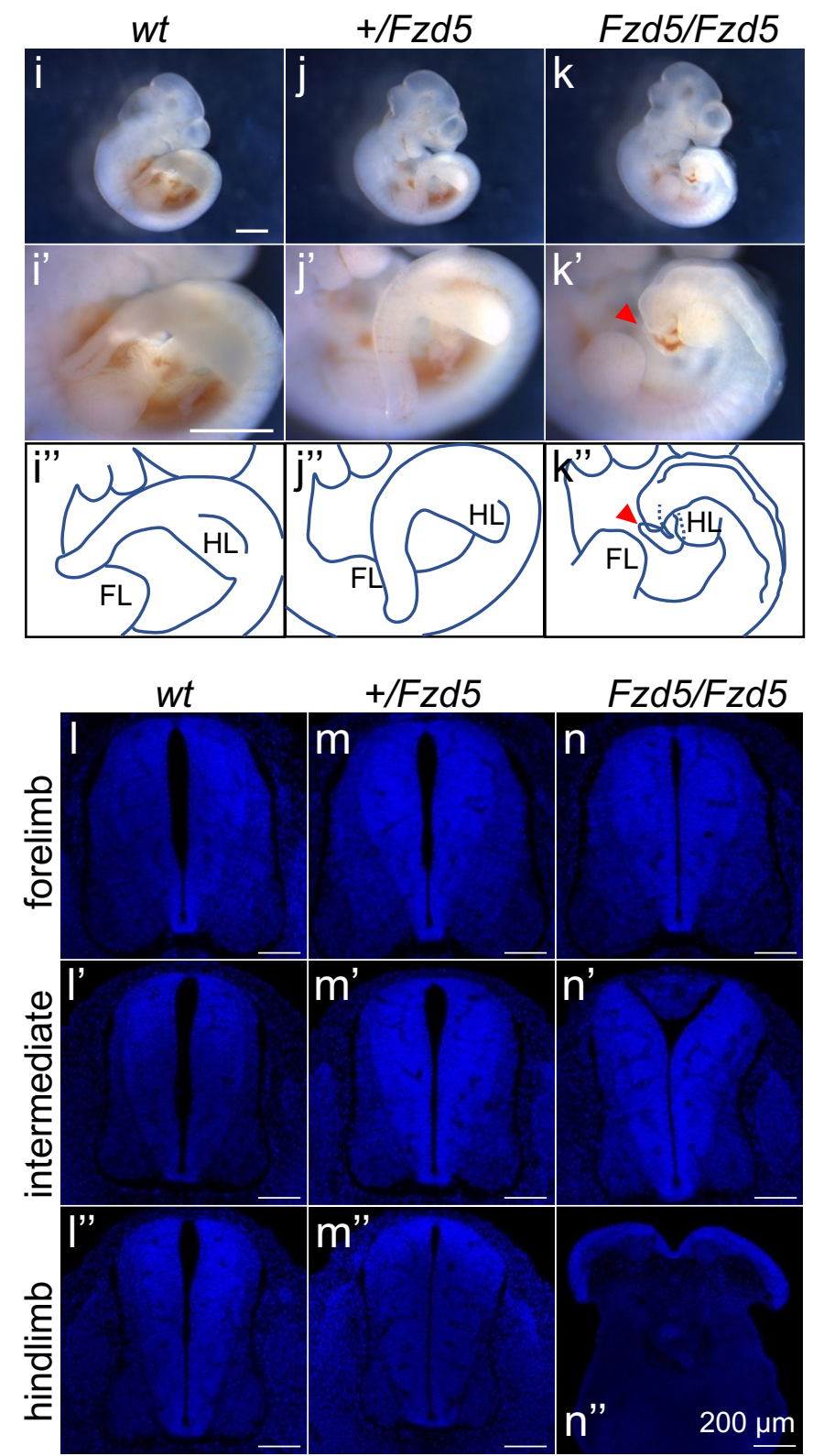
Fig. 2

E10.5

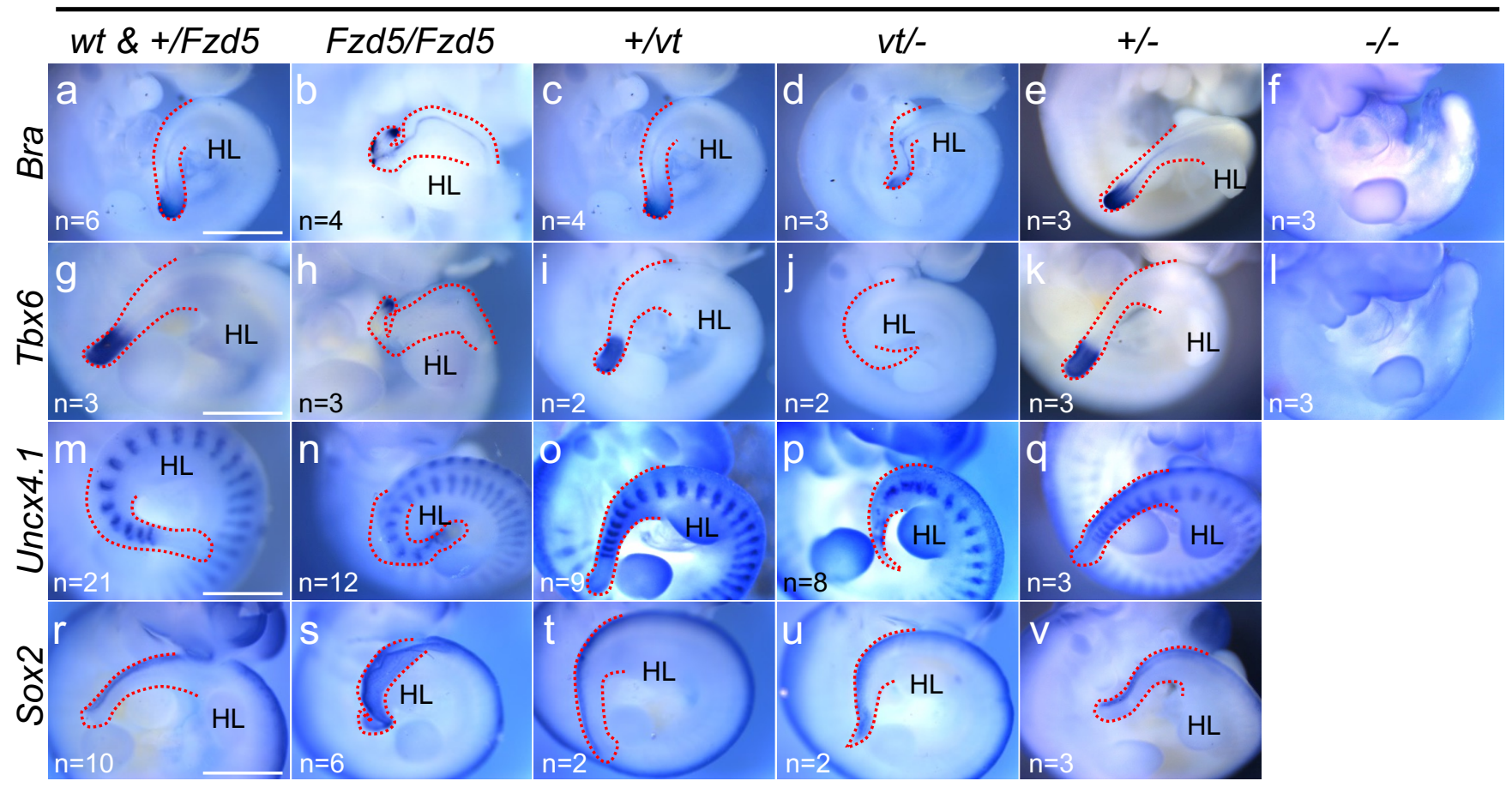

E12.5

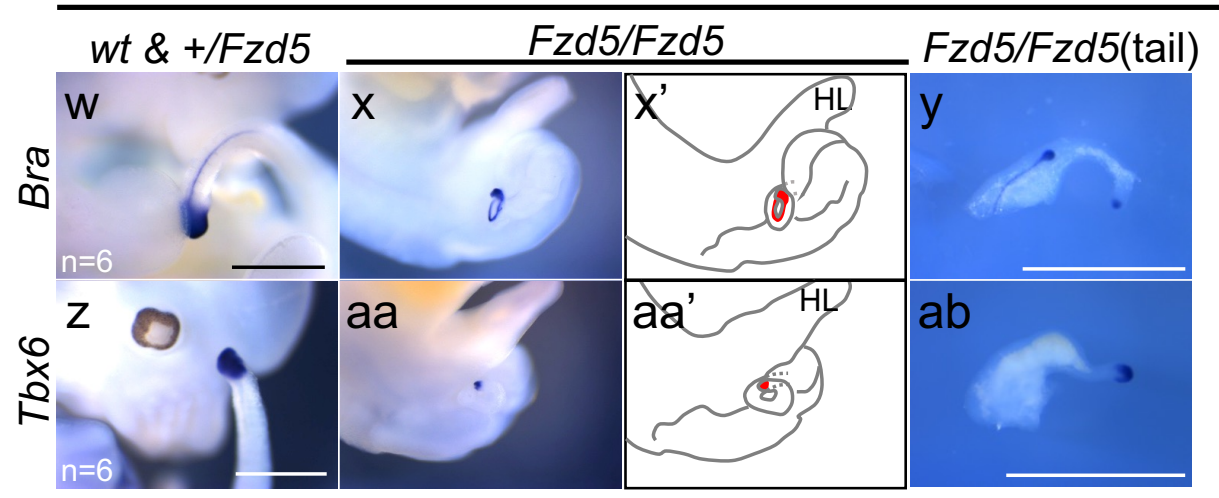



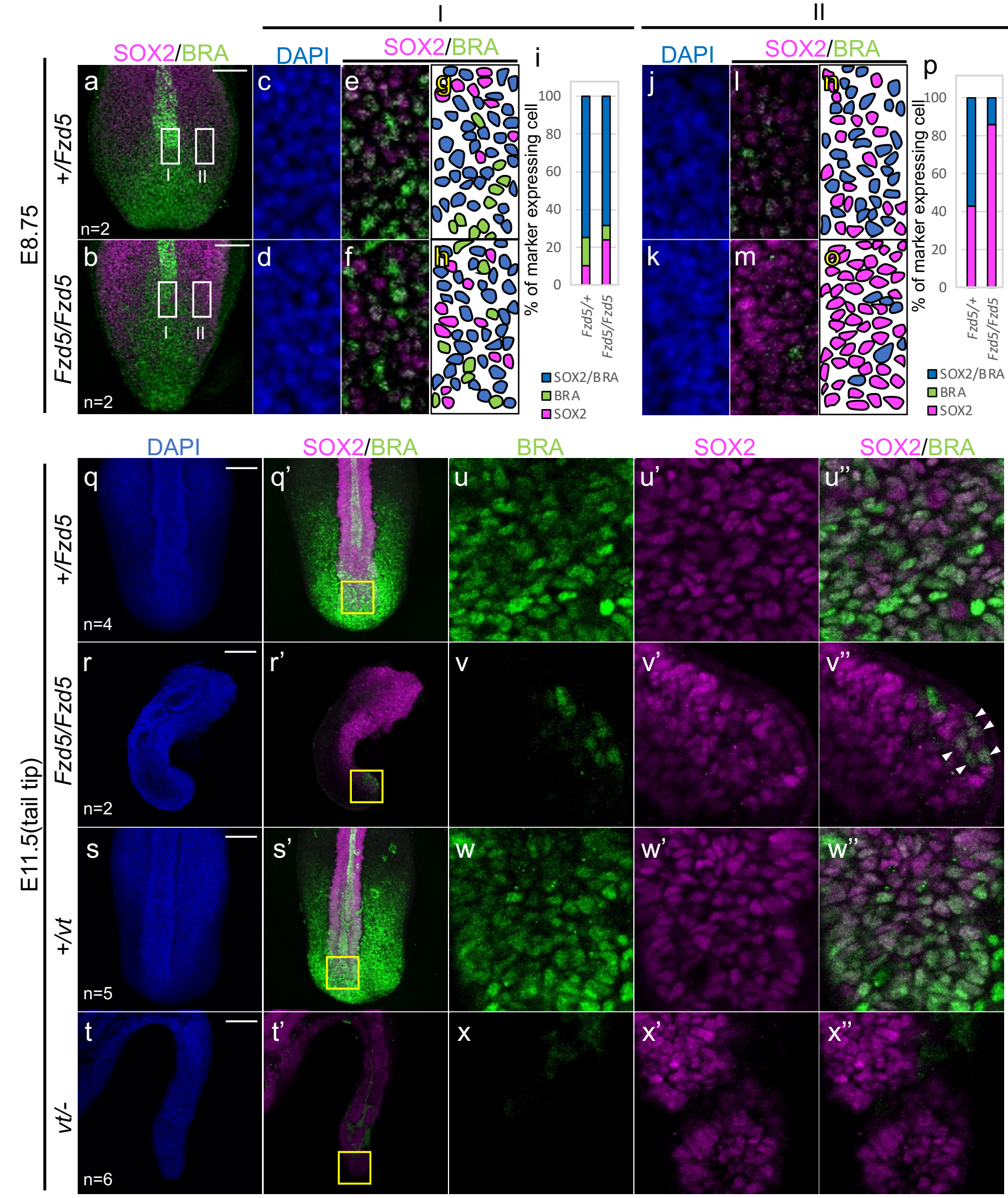
Fig. 5
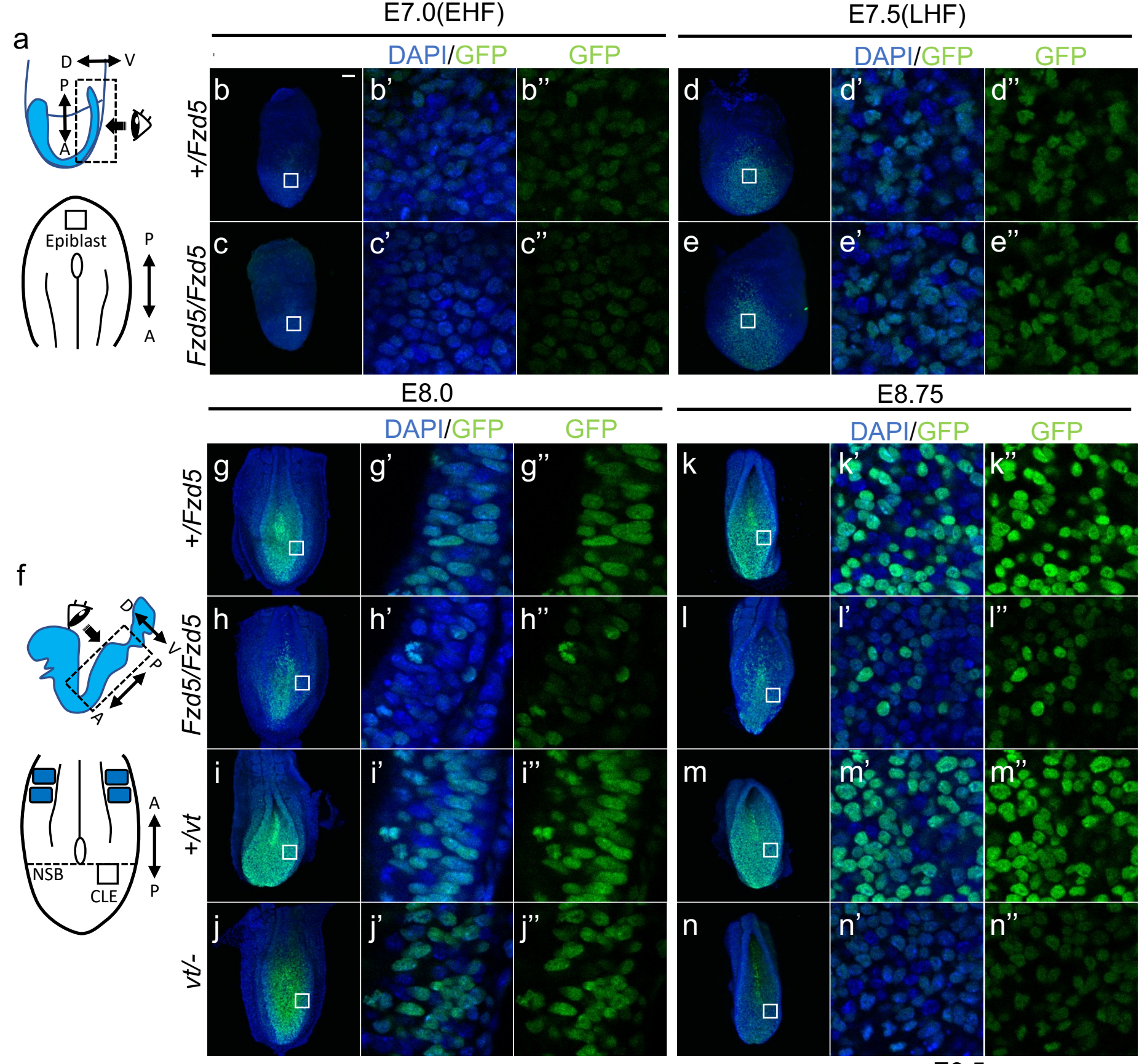

E8.75
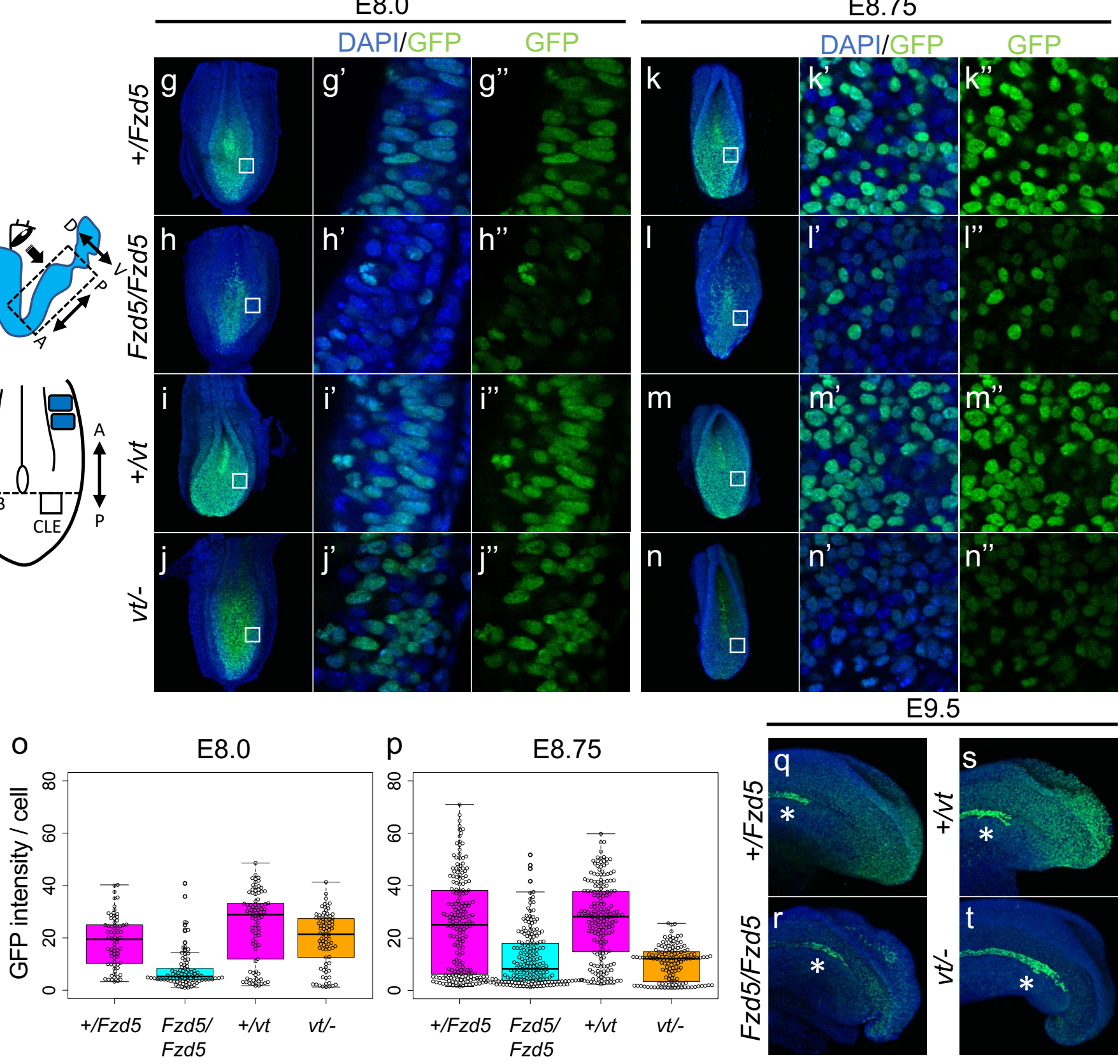
Fig. 6

a

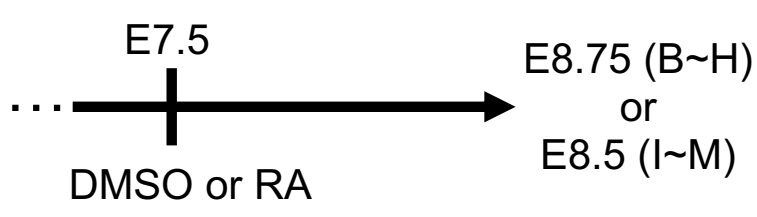

b

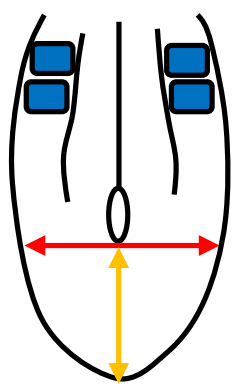

Width at NSB

Length posterior to NSB
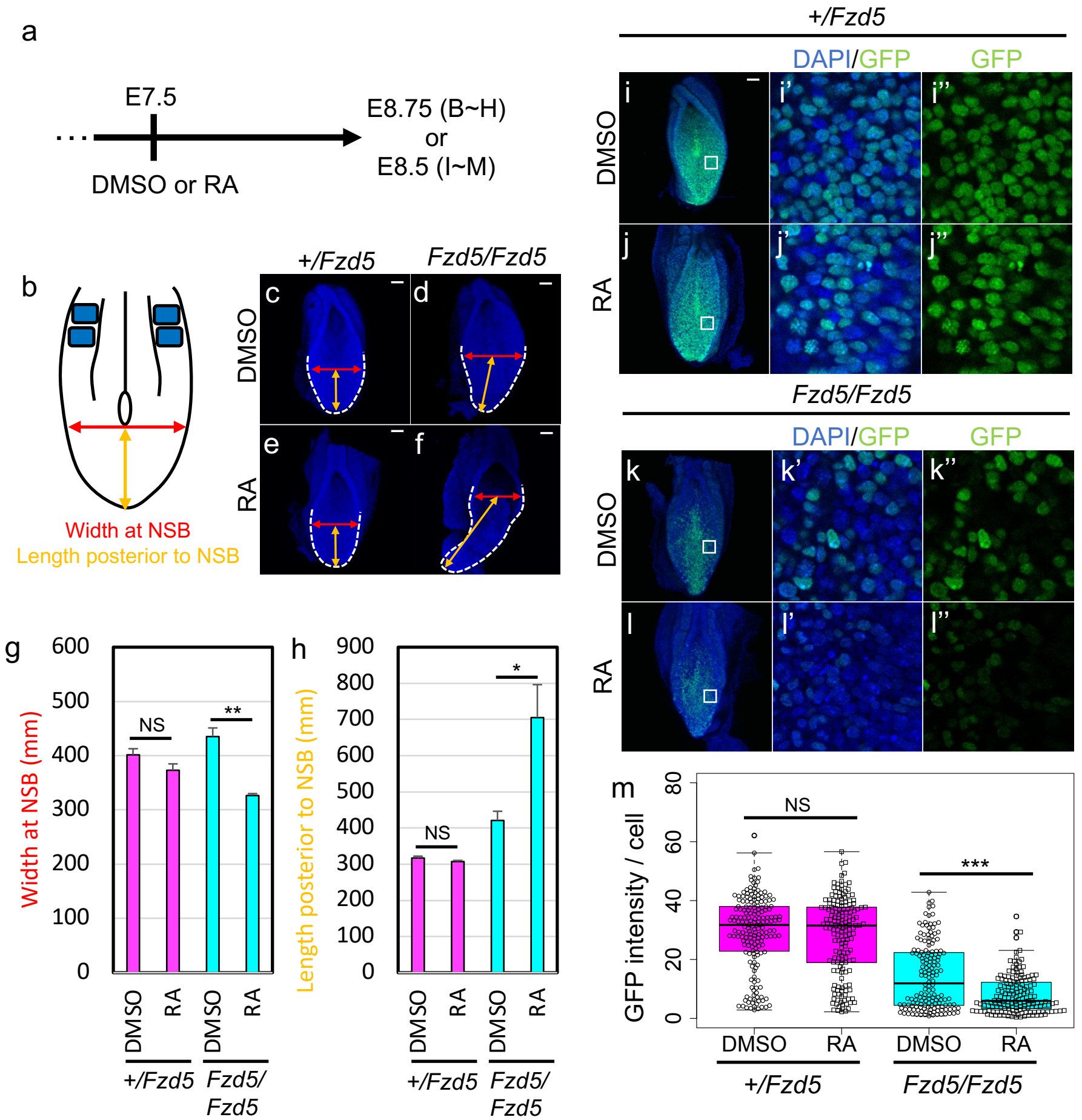
a

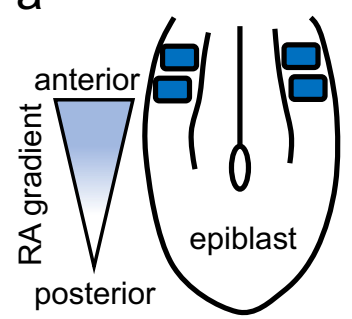

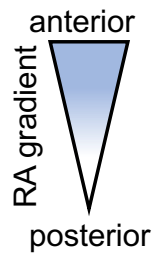

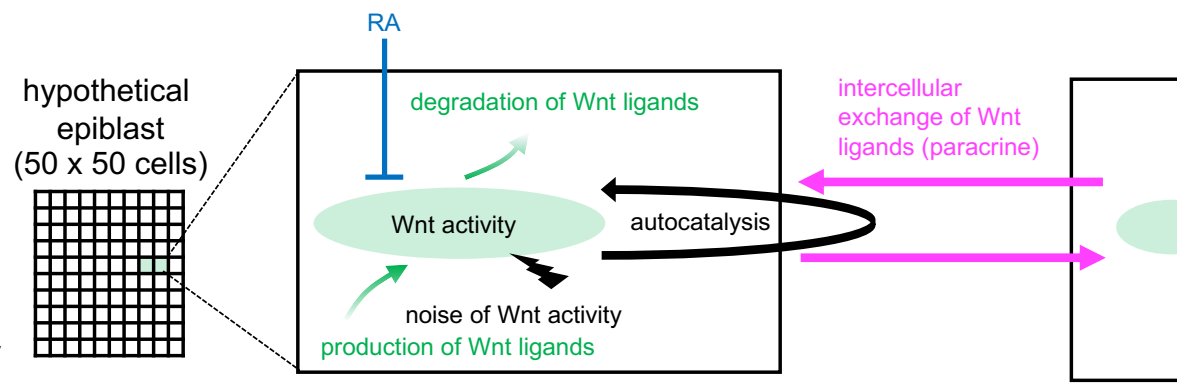

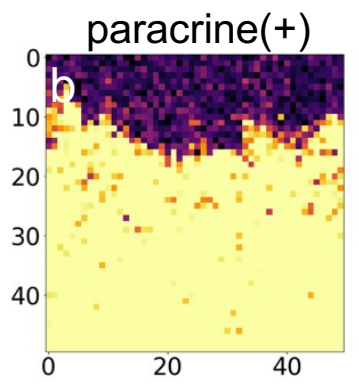

g
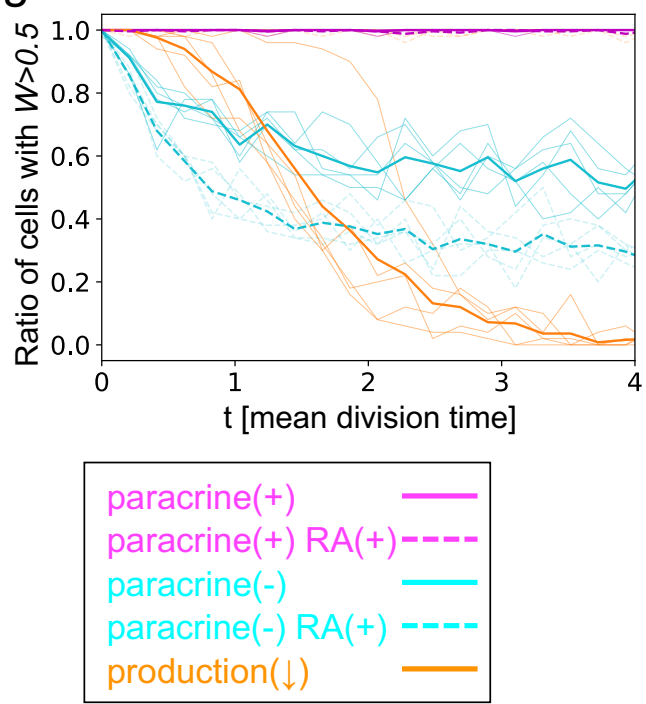
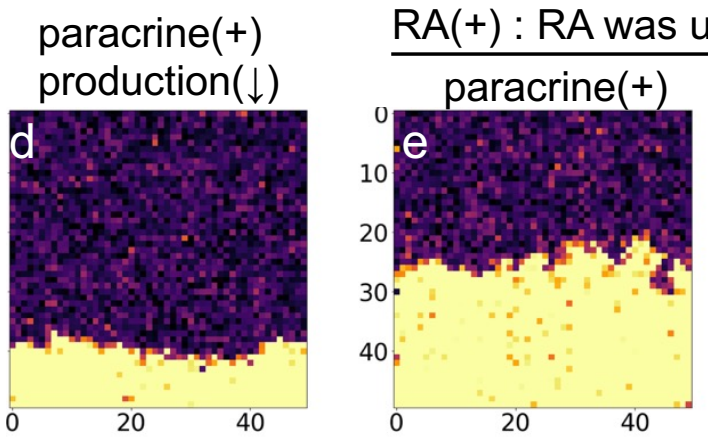

h

E7.0

E8.0

\section{E8.75}

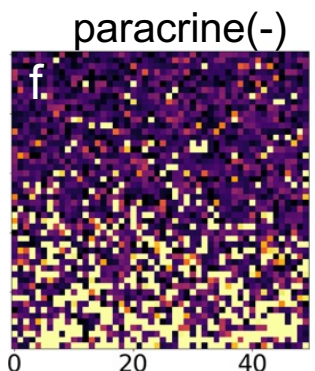

40

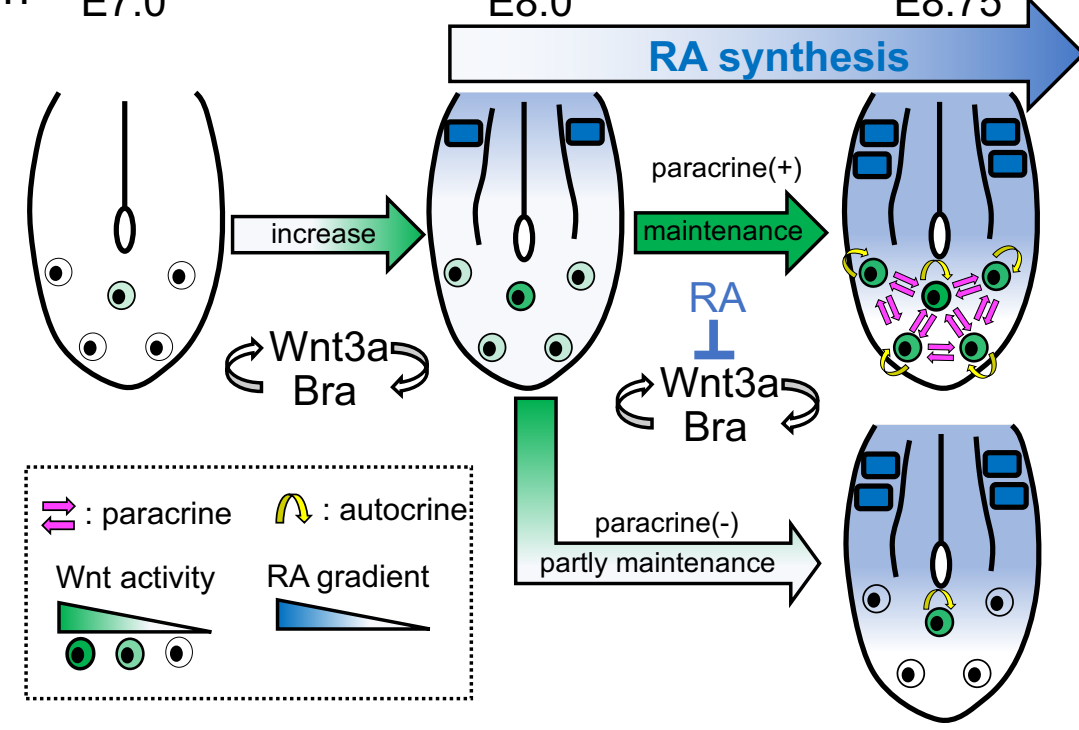




\section{Supplementary Files}

This is a list of supplementary files associated with this preprint. Click to download.

- aDiffusionRA.mp4

- NCBformatExtendedDataFig..pdf

- dDiffusionRA.mp4

- cProduction.mp4

- bDiffusionRA.mp4

- eDiffusionRA.mp4 